Supporting Information (SI) for:

\title{
The Thermochemistry and Initial Decomposition Pathways of Triazole Energetic Materials
}

Meiheng Lu, ${ }^{\text {ab }}$ Panwang Zhou, ${ }^{* c}$ Yanqiang Yang, ${ }^{\mathrm{d}}$ Jianyong Liu*b, Bing Jin ${ }^{\mathrm{e}}$, Keli Han ${ }^{\mathrm{b}}$

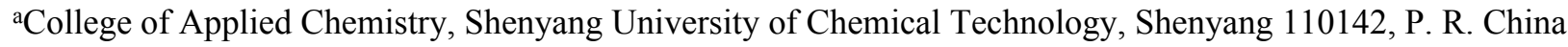

bState Key Laboratory of Molecular Reaction Dynamics, Dalian Institute of Chemical Physics, Chinese Academy of Sciences, Dalian 116023, P. R. China

'Institute of Molecular Sciences and Engineering, Shandong University, Qingdao 266235, P. R. China

dNational Key Laboratory of Shock Wave and Detonation Physics, Institute of Fluid Physics, China Academy of Engineering Physics, Chengdu 610200, P. R. China

eState Key Laboratory of Magnetic Resonance and Atomic and Molecular Physics, Wuhan Institute of Physics and Mathematics, Chinese Academy of Sciences, Wuhan 430071, P. R. China

Corresponding Author:

*Jianyong Liu: beam@dicp.ac.cn

*Panwang Zhou:pwzhou@sdu.edu.cn 


\section{T1 values for critical structures}

\begin{tabular}{|c|c|c|c|c|c|c|c|}
\hline Reactants & T1 value & $\begin{array}{c}\begin{array}{c}\text { Transition } \\
\text { States }\end{array} \\
\end{array}$ & T1 value & $\begin{array}{c}\text { Intermedi } \\
\text { ates }\end{array}$ & T1 value & Products & T1 value \\
\hline $\mathrm{R} 1$ & 0.0134 & TS1 & 0.0159 & IM1 & 0.0153 & P1 & 0.0125 \\
\hline $\mathrm{R} 2$ & 0.0125 & $\mathrm{TS} 2$ & 0.0171 & IM2 & 0.0139 & $\mathrm{P} 2$ & 0.0190 \\
\hline $\mathrm{R} 3$ & 0.0119 & $\mathrm{TS} 3$ & 0.0194 & IM3 & 0.0185 & $\mathrm{P} 3$ & 0.0217 \\
\hline $\mathrm{R} 4$ & 0.0112 & TS4 & 0.0150 & IM4 & 0.0185 & $\mathrm{P} 4$ & 0.0142 \\
\hline $\mathrm{R} 5$ & 0.0156 & TS5 & 0.0174 & IM5 & 0.0178 & P5 & 0.0160 \\
\hline R6 & 0.0159 & TS6 & 0.0239 & IM6 & 0.0139 & P6 & 0.0217 \\
\hline R7 & 0.0159 & TS7 & 0.0182 & IM7 & 0.0141 & $\mathrm{P} 7$ & 0.0129 \\
\hline $\mathrm{R} 8$ & 0.0158 & TS8 & 0.0168 & IM8 & 0.0184 & P8 & 0.0141 \\
\hline R9 & 0.0162 & TS9 & 0.0219 & IM9 & 0.0164 & P9 & 0.0207 \\
\hline \multirow[t]{22}{*}{ R10 } & 0.0156 & TS10 & 0.0231 & IM10 & 0.0170 & P10 & 0.0119 \\
\hline & & TS11 & 0.0122 & IM11 & 0.0178 & P11 & 0.0196 \\
\hline & & $\mathrm{TS} 12$ & 0.0174 & IM12 & 0.0151 & $\mathrm{P} 12$ & 0.0211 \\
\hline & & TS13 & 0.0126 & IM13 & 0.0168 & $\mathrm{P} 13$ & 0.0219 \\
\hline & & TS14 & 0.0214 & IM14 & 0.0178 & $\mathrm{P} 14$ & 0.0201 \\
\hline & & TS15 & 0.0200 & IM15 & 0.0168 & $\mathrm{P} 15$ & 0.0267 \\
\hline & & TS16 & 0.0189 & IM16 & 0.0173 & $\mathrm{P} 16$ & 0.0180 \\
\hline & & TS17 & 0.0239 & IM17 & 0.0153 & P17 & 0.0237 \\
\hline & & TS18 & 0.0185 & IM18 & 0.0170 & $\mathrm{P} 18$ & 0.0220 \\
\hline & & TS19 & 0.0208 & IM19 & 0.0169 & P19 & 0.0197 \\
\hline & & TS20 & 0.0135 & IM20 & 0.0156 & $\mathrm{P} 20$ & 0.0237 \\
\hline & & TS21 & 0.0169 & IM21 & 0.0180 & $\mathrm{P} 21$ & 0.0236 \\
\hline & & TS22 & 0.0184 & IM22 & 0.0165 & $\mathrm{P} 22$ & 0.0201 \\
\hline & & TS23: & 0.0183 & IM23 & 0.0168 & $\mathrm{P} 23$ & 0.0277 \\
\hline & & TS24 & 0.0155 & IM24 & 0.0167 & $\mathrm{P} 24$ & 0.0181 \\
\hline & & TS25 & 0.0170 & IM25 & 0.0180 & $\mathrm{P} 25$ & 0.0172 \\
\hline & & TS26 & 0.0221 & IM26 & 0.0167 & P26 & 0.0238 \\
\hline & & $\mathrm{TS} 27$ & 0.0186 & & & $\mathrm{P} 27$ & 0.0250 \\
\hline & & TS28 & 0.0161 & & & $\mathrm{P} 28$ & 0.0249 \\
\hline & & TS29 & 0.0221 & & & P29 & 0.0196 \\
\hline & & TS30 & 0.0181 & & & P30-1 & 0.0190 \\
\hline & & TS31 & 0.0159 & & & P30-2 & 0.0188 \\
\hline
\end{tabular}




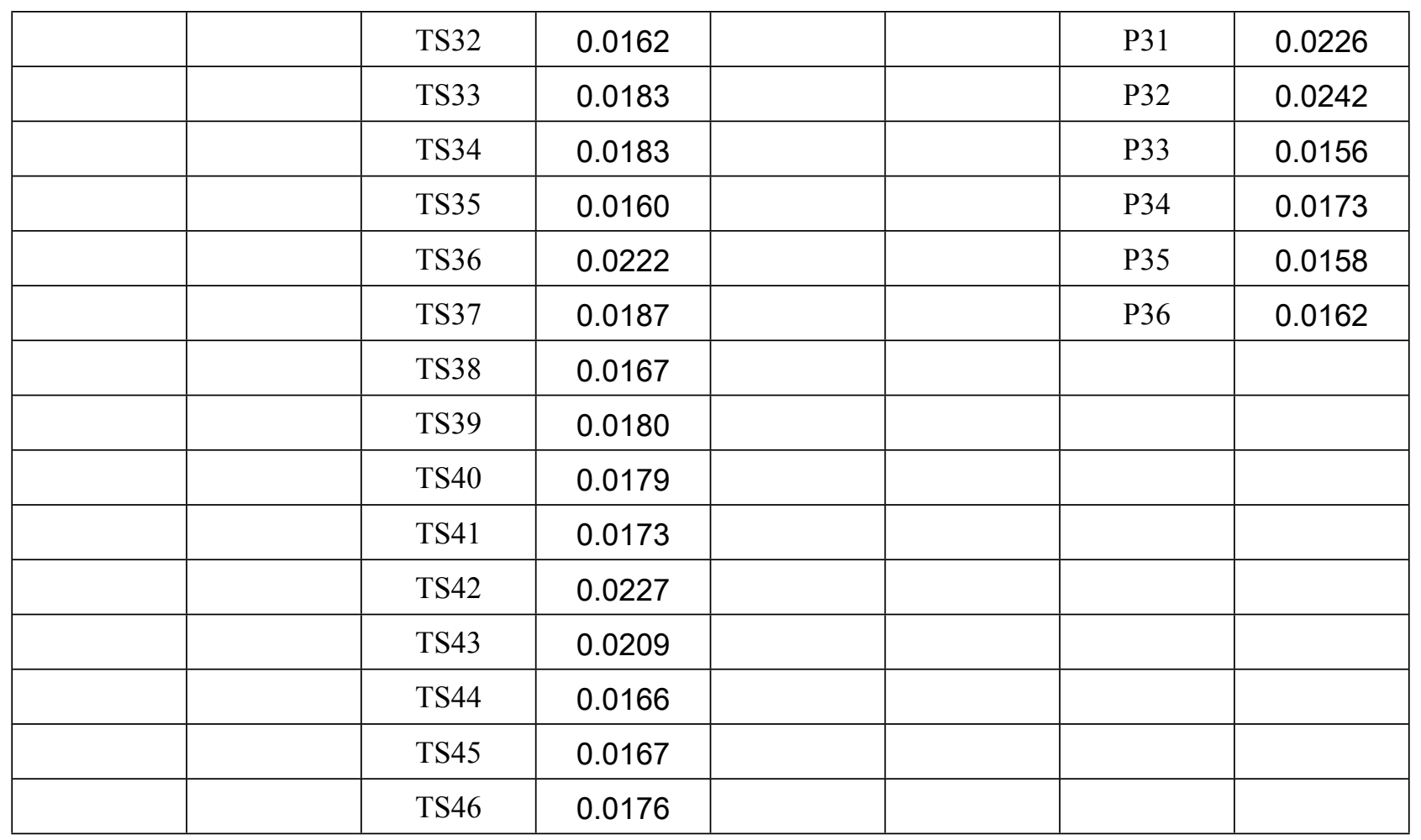

The cartesian coordinates of critical structures

\section{Reactants:}

R1

$\mathrm{C}$

$\mathrm{H}$

$\mathrm{H}$

$\mathrm{N}$

C

$\mathrm{N}$

$\mathrm{H}$

$\mathrm{N}$

$\mathrm{R} 2$

$\mathrm{C}$

$\mathrm{H}$

$\mathrm{H}$

$\mathrm{H}$

$\mathrm{N}$

$\mathrm{N}$

C

N

R3

C

$\mathrm{N}$

$\mathrm{H}$

$\mathrm{H}$

$\mathrm{H}$

N

$$
\begin{array}{rrr}
-1.12186700 & 0.01058800 & -0.00001100 \\
1.74002700 & -1.32988700 & -0.00004800 \\
-2.20133500 & 0.04804600 & 0.00004900 \\
-0.38760500 & 1.13035400 & -0.00016200 \\
0.84670400 & -0.72220800 & -0.00009900 \\
0.86370800 & 0.63332100 & -0.00000700 \\
1.65350700 & 1.26377400 & 0.00028700 \\
-0.41056300 & -1.15113400 & 0.00022200
\end{array}
$$

$\begin{array}{rrc}1.08229500 & 0.25888700 & 0.00030100 \\ -2.10658200 & 0.60042100 & 0.00013900 \\ 2.10625700 & 0.60152500 & 0.00040200 \\ -0.00055700 & 2.10122500 & -0.00038700 \\ -0.68941700 & -1.00349600 & 0.00016900 \\ 0.68996200 & -1.00314900 & -0.00031200 \\ -1.08244100 & 0.25832300 & 0.00008200 \\ -0.00029400 & 1.09144000 & -0.00020800\end{array}$

$$
\begin{array}{rrr}
-1.05248200 & 0.47818600 & -0.00013100 \\
0.16702200 & 1.13386000 & 0.00012100 \\
1.06366300 & 0.11600200 & 0.00005500 \\
2.07388300 & 0.15841700 & 0.00008300 \\
-2.04962800 & 0.89114300 & -0.00022300 \\
0.45457800 & 2.17335400 & 0.00021600 \\
0.46595100 & -1.09155100 & -0.00035100
\end{array}
$$




\section{R4}

$\begin{array}{lrrr}\text { C } & 0.91966100 & 0.69676000 & -0.00002400 \\ \mathrm{C} & 0.91433300 & -0.70324600 & 0.00007000 \\ \mathrm{H} & 1.74160100 & 1.39654500 & -0.00005600 \\ \mathrm{H} & 1.73109000 & -1.40896200 & 0.00013700 \\ \mathrm{~N} & -0.35751700 & 1.13800000 & -0.00009100 \\ \mathrm{~N} & -1.04995300 & 0.00367200 & -0.00004100 \\ \mathrm{H} & -2.06114000 & 0.00736600 & -0.00009200 \\ \mathrm{~N} & -0.36617500 & -1.13539100 & 0.00009500\end{array}$

\section{R5}

R6

$\begin{array}{cccc}\mathrm{C} & 1.98501600 & 0.69498800 & -0.12638200 \\ \mathrm{H} & 2.88855100 & 1.27691600 & -0.23935400 \\ \mathrm{~N} & 0.73655900 & 1.14615500 & -0.21074400 \\ \mathrm{~N} & 0.71629500 & -1.10908300 & 0.20111000 \\ \mathrm{~N} & 1.97327800 & -0.63835200 & 0.12106200 \\ \mathrm{H} & 2.75036200 & -1.27378100 & 0.25086000 \\ \mathrm{C} & 0.02881400 & 0.01688400 & -0.00902600 \\ \mathrm{~N} & -1.42990300 & 0.00106300 & -0.00222900 \\ \mathrm{O} & -1.98862900 & 1.05596600 & 0.29379200 \\ \mathrm{O} & -1.97330800 & -1.06507200 & -0.28922300\end{array}$

\section{R7}

$\begin{array}{lcrc}\mathrm{C} & -2.06765100 & 0.55991700 & 0.00011200 \\ \mathrm{H} & -2.94260600 & 1.19311700 & 0.00022400 \\ \mathrm{H} & -0.44140500 & 1.98310300 & 0.00032900 \\ \mathrm{~N} & -0.76351400 & -1.18984000 & -0.00022200 \\ \mathrm{~N} & -2.07156900 & -0.76610500 & -0.00015700 \\ \mathrm{C} & -0.03220700 & -0.09352200 & -0.00000800 \\ \mathrm{~N} & -0.78720900 & 1.03145400 & 0.00023600 \\ \mathrm{~N} & 1.41610900 & -0.01255100 & 0.00001100 \\ \mathrm{O} & 2.04926000 & -1.06083500 & 0.00030800 \\ \mathrm{O} & 1.87904500 & 1.13392400 & -0.00034000\end{array}$

R8

$\begin{array}{cccc}\mathrm{C} & 0.89934300 & 1.15059600 & 0.00000000 \\ \mathrm{C} & 0.00805200 & 0.08970900 & 0.00005700 \\ \mathrm{~N} & 0.77592300 & -1.01746200 & 0.00025300 \\ \mathrm{H} & 0.47533800 & -1.98615400 & 0.00045100 \\ \mathrm{H} & 0.69973500 & 2.21081200 & -0.00007100 \\ \mathrm{~N} & 2.08357700 & -0.69390500 & 0.00003500 \\ \mathrm{~N} & 2.15325000 & 0.63304500 & -0.00020200 \\ \mathrm{~N} & -1.42942700 & 0.01700300 & 0.00003000\end{array}$


$\begin{array}{llll}\mathrm{O} & -1.92394300 & -1.11384700 & 0.00002600\end{array}$

$\begin{array}{lllll}\mathrm{O} & -2.03889500 & 1.08419100 & -0.00021900\end{array}$

R9

$\begin{array}{llll}\mathrm{C} & -0.82820400 & 1.10026700 & 0.00017500\end{array}$

$\begin{array}{llll}\mathrm{C} & -0.00668200 & -0.01185400 & 0.00001000\end{array}$

$\begin{array}{llll}\mathrm{H} & -0.63936300 & 2.16136900 & 0.00028400\end{array}$

$\mathrm{N} \quad-2.00558600 \quad-0.81409400 \quad-0.00002900$

$\begin{array}{llll}\mathrm{N} & 1.44667100 & 0.00156700 & -0.00004200\end{array}$

$\begin{array}{llll}\mathrm{O} & 2.03318100 & -1.07513000 & -0.00018600\end{array}$

$\begin{array}{llll}\mathrm{O} & 1.96741100 & 1.11955700 & 0.00002000\end{array}$

$\mathrm{N} \quad-0.72577400 \quad-1.15329200 \quad-0.00004000$

$\begin{array}{llll}\mathrm{N} & -2.05711200 & 0.53264100 & 0.00008500\end{array}$

$\begin{array}{llll}\mathrm{H} & -2.96344500 & 0.98498700 & 0.00012300\end{array}$

\section{R10}

$\begin{array}{llll}\mathrm{C} & 0.83789900 & 1.13238200 & -0.00140600\end{array}$

$\begin{array}{lllll}\mathrm{C} & 0.00686700 & 0.00889000 & -0.00007000\end{array}$

$\mathrm{H} \quad 0.59557800 \quad 2.18311300 \quad-0.00244100$

$\mathrm{N} \quad-1.44444500 \quad-0.01859800 \quad-0.00000900$

$\begin{array}{llll}\mathrm{O} & -2.00261200 & -1.11175300 & -0.00154200\end{array}$

$\begin{array}{llll}\mathrm{O} & -1.99348200 & 1.08567300 & 0.00176500\end{array}$

$\begin{array}{llll}\mathrm{N} & 0.73026500 & -1.12132100 & 0.00088000\end{array}$

$\mathrm{N} \quad \begin{array}{llll}\mathrm{N} & 2.11204800 & 0.68925500 & -0.00045600\end{array}$

$\begin{array}{llll}\mathrm{N} & 1.96466000 & -0.63116900 & 0.00074000\end{array}$

$\mathrm{H} \quad 2.76687400 \quad-1.24927700 \quad 0.00143000$ 


\section{Transition States:}

$\begin{array}{lrrr}\text { TS1 } & & & \\ \text { C } & -0.89157700 & 0.67534500 & 0.02434500 \\ \mathrm{H} & -0.59396900 & -1.57123900 & 0.69723000 \\ \mathrm{H} & -1.75397400 & 1.32522100 & 0.04026800 \\ \mathrm{~N} & 0.34871000 & 1.13499800 & -0.00118800 \\ \mathrm{C} & 0.37692500 & -1.15251200 & -0.07786400 \\ \mathrm{~N} & 1.08893200 & -0.00316700 & 0.02218000 \\ \mathrm{H} & 2.09672000 & 0.07841300 & 0.02739900 \\ \mathrm{~N} & -0.96062300 & -0.69888700 & -0.08439000\end{array}$

$\begin{array}{lcrc}\text { TS2 } & & & \\ \mathrm{C} & 0.97058800 & -0.51787000 & 0.00986900 \\ \mathrm{H} & 1.42789900 & 1.56141800 & 0.03652300 \\ \mathrm{H} & 1.96021100 & -0.95115200 & -0.03182000 \\ \mathrm{~N} & -0.14378800 & -1.20956000 & 0.02481700 \\ \mathrm{C} & -0.62085300 & 1.04212100 & -0.08791300 \\ \mathrm{~N} & -1.15319800 & -0.24231000 & -0.08622200 \\ \mathrm{H} & -1.51278700 & 0.58588500 & 0.75703300 \\ \mathrm{~N} & 0.72931000 & 0.83163400 & 0.01948100\end{array}$

\section{TS3}

$\begin{array}{lrrc}\mathrm{C} & -0.77747300 & -1.12741800 & 0.03896100 \\ \mathrm{H} & 1.91950900 & 1.32359900 & -0.02974700 \\ \mathrm{H} & -1.25572500 & -2.08321700 & -0.10993800 \\ \mathrm{~N} & -1.15538500 & 0.03547400 & 0.00114700 \\ \mathrm{C} & 1.32500400 & 0.43042600 & 0.00255800 \\ \mathrm{~N} & -0.59744200 & 1.18791200 & -0.12676000 \\ \mathrm{H} & -0.84626100 & 1.78092600 & 0.67192800 \\ \mathrm{~N} & 1.30958300 & -0.77186500 & 0.01399000\end{array}$

\section{TS4}

$\mathrm{C}$

$\mathrm{H}$

$\mathrm{H}$

$\mathrm{N}$

C

$\mathrm{N}$

$\mathrm{H}$

N

\section{TS5}

$\mathrm{C}$

$\mathrm{H}$

$\mathrm{H}$

$\mathrm{N}$

C

$\mathrm{N}$

H

N $\begin{array}{ccc}1.07140800 & 0.30441300 & -0.00621000 \\ -2.01419100 & 0.75535500 & -0.28912000 \\ 2.12021100 & 0.57045800 & 0.01756300 \\ 0.67441600 & -0.99283400 & 0.00006200 \\ -1.01525500 & 0.42699700 & -0.02595700 \\ -0.65654600 & -0.98568800 & -0.07894600 \\ -1.15632100 & -0.34619900 & 0.95645200 \\ 0.08404200 & 1.21165500 & 0.00861300\end{array}$

$$
\begin{array}{rcc}
-0.90109900 & 1.01151200 & -0.00019100 \\
-0.20413700 & -1.79345900 & -0.91621800 \\
-1.56766000 & 1.86975900 & 0.00038600 \\
1.12072500 & 0.88530400 & -0.00009300 \\
-0.22753000 & -1.20247600 & -0.00014000 \\
1.29530800 & -0.27896800 & 0.00019400 \\
-0.20400100 & -1.79415800 & 0.91546600 \\
-1.16638100 & -0.19724400 & 0.00023500
\end{array}
$$




$\begin{array}{lrrr}\text { TS6 } & & & \\ \mathrm{C} & -1.10366500 & -1.01085000 & -0.00879200 \\ \mathrm{H} & 0.13485000 & 2.05733000 & -0.40611600 \\ \mathrm{H} & -2.14746300 & -1.35604400 & -0.04006700 \\ \mathrm{H} & -1.94502000 & 0.86840100 & -0.00216900 \\ \mathrm{~N} & 1.11080700 & 0.25042100 & -0.01744000 \\ \mathrm{~N} & 1.42761600 & -0.85096100 & -0.00384500 \\ \mathrm{C} & 0.09059100 & 1.11164900 & 0.11133700 \\ \mathrm{~N} & -1.10469700 & 0.28990100 & -0.00256000\end{array}$

$\begin{array}{lrrr}\text { TS7 } & & & \\ \mathrm{C} & 1.91869100 & -0.51470500 & -0.45432800 \\ \mathrm{H} & 0.02987600 & 1.44725800 & -0.93759800 \\ \mathrm{H} & 2.74596200 & -0.89957000 & 0.17584700 \\ \mathrm{H} & 1.29934500 & 0.20349500 & 1.38182700 \\ \mathrm{~N} & -1.15960200 & 0.05594700 & -0.06259100 \\ \mathrm{~N} & -2.13164900 & -0.53357000 & 0.03568700 \\ \mathrm{C} & -0.05937300 & 0.81655100 & -0.06320200 \\ \mathrm{~N} & 1.11538100 & 0.11158600 & 0.38191900\end{array}$

\section{TS8}

$\begin{array}{llll}\text { C } & -2.09197900 & -0.39763500 & 0.47690000\end{array}$

$\begin{array}{llll}\mathrm{H} & -0.30386500 & 1.14978100 & 0.98416200\end{array}$

$\begin{array}{llll}\mathrm{H} & -2.82995000 & -0.98070000 & -0.11210600\end{array}$

$\begin{array}{llll}\mathrm{H} & -1.21567500 & -0.24346900 & -1.39165500\end{array}$

$\begin{array}{llll}\mathrm{N} & 1.32736300 & -0.07957200 & 0.18943700\end{array}$

$\begin{array}{llll}\mathrm{N} & 2.39115800 & -0.36648800 & -0.05013500\end{array}$

$\begin{array}{lllll}\mathrm{C} & -0.15826300 & 0.93772700 & -0.08587300\end{array}$

$\begin{array}{llll}\mathrm{N} & -1.16838600 & -0.00624800 & -0.40024000\end{array}$

\section{TS9}

$\begin{array}{lrrr}\mathrm{C} & -1.39222600 & 0.64722900 & -0.05653200 \\ \mathrm{H} & 2.09448500 & -0.72912900 & -0.04706900 \\ \mathrm{H} & -2.08940800 & 1.43819100 & 0.12619700 \\ \mathrm{H} & 1.75958600 & 1.54970200 & -0.05837100 \\ \mathrm{~N} & 0.08599000 & -1.20183500 & 0.00215000 \\ \mathrm{~N} & -0.99607700 & -0.47077000 & 0.02090200 \\ \mathrm{C} & 1.11385900 & -0.25908000 & -0.00279100 \\ \mathrm{~N} & 0.89659200 & 1.01722500 & 0.02483100\end{array}$

\section{TS10}

$\begin{array}{lrrr}\mathrm{C} & 2.34423300 & -0.33107200 & 0.00223100 \\ \mathrm{H} & -1.89693300 & 1.26652700 & 0.00502600 \\ \mathrm{H} & 3.37044700 & -0.62962000 & 0.00754500 \\ \mathrm{H} & -2.67093400 & -1.02675500 & 0.00261300 \\ \mathrm{~N} & -0.29214800 & 1.08285100 & -0.00005400 \\ \mathrm{~N} & 1.21656500 & -0.02885600 & -0.00444700 \\ \mathrm{C} & -1.28979600 & 0.27697100 & 0.00105800 \\ \mathrm{~N} & -1.65716000 & -0.95193000 & -0.00048800\end{array}$




$\begin{array}{lrrr}\text { TS11 } & & & \\ \text { C } & -1.06002200 & -0.33909700 & -0.01497700 \\ \text { H } & 2.08551300 & -0.52664000 & -0.28217300 \\ \text { H } & -2.08640300 & -0.67421200 & 0.04146100 \\ \text { H } & 0.72736200 & -0.93240100 & 0.96693000 \\ \text { N } & 0.62147900 & 1.03498400 & 0.00586900 \\ \text { N } & -0.71983000 & 0.96602800 & 0.00191600 \\ \text { C } & 1.07340500 & -0.24275900 & -0.02310300 \\ \text { N } & -0.01690200 & -1.19752800 & -0.07889100\end{array}$

\section{TS12}

$\begin{array}{lrrr}\mathrm{C} & 0.90181300 & 1.01073100 & 0.00001900 \\ \mathrm{H} & 0.20176700 & -1.79293700 & 0.91662100 \\ \mathrm{H} & 1.56881400 & 1.86863900 & 0.00018400 \\ \mathrm{H} & 0.20224600 & -1.79487100 & -0.91501200 \\ \mathrm{~N} & -1.29515800 & -0.27787700 & -0.00031600 \\ \mathrm{~N} & -1.11962700 & 0.88614700 & 0.00015600 \\ \mathrm{C} & 0.22613700 & -1.20257000 & 0.00017900 \\ \mathrm{~N} & 1.16613800 & -0.19824100 & -0.00026500\end{array}$

\section{TS13}

$\mathrm{C}$

$\mathrm{C}$

$\mathrm{N}$

$\mathrm{H}$

$\mathrm{H}$

$\mathrm{H}$

$\mathrm{N}$

$\mathrm{N}$

\section{TS14}

$\begin{array}{lrrr}\mathrm{C} & -1.11591100 & 0.07293000 & -0.00002000 \\ \mathrm{C} & -0.47290900 & -1.23586500 & 0.00014700 \\ \mathrm{~N} & 1.35181600 & -0.46377200 & -0.00034200 \\ \mathrm{H} & -0.62390300 & -1.81700000 & 0.91465300 \\ \mathrm{H} & -2.21833400 & -0.00320300 & -0.00064700 \\ \mathrm{H} & -0.62458300 & -1.81770000 & -0.91378700 \\ \mathrm{~N} & 1.29525200 & 0.68814300 & 0.00021400 \\ \mathrm{~N} & -0.78996200 & 1.29213100 & -0.00001200\end{array}$

\section{TS15}

$\begin{array}{lrrr}\mathrm{C} & -1.15144400 & 0.25157100 & 0.00005800 \\ \mathrm{C} & -1.22953500 & -1.19190100 & 0.00007100 \\ \mathrm{~N} & 1.84374000 & -0.67004300 & -0.00008800 \\ \mathrm{H} & -1.63246300 & -1.60463900 & 0.92429000 \\ \mathrm{H} & -2.29071000 & 0.23203200 & 0.00012600 \\ \mathrm{H} & -1.63257300 & -1.60465100 & -0.92409400 \\ \mathrm{~N} & 1.88586300 & 0.45196700 & -0.00010300 \\ \mathrm{~N} & -0.89508600 & 1.44939500 & 0.00003500\end{array}$

$\begin{array}{lll}0.91282500 & 0.69483500 & 0.00107900\end{array}$

$\begin{array}{lll}-0.44231600 & 1.04648000 & -0.02697200\end{array}$

$-1.16194000 \quad-0.22242300 \quad-0.07975100$

$\begin{array}{lll}-1.07705500 & 0.52429300 & 0.95066700\end{array}$

$\begin{array}{lll}-0.95377000 & 1.97032700 & -0.26191700\end{array}$

$\begin{array}{lll}-0.22151400 & -1.17116900 & 0.00088000\end{array}$

$\begin{array}{lll}1.01501400 & -0.64518400 & -0.00294400\end{array}$ $\begin{array}{lll}1.78685000 & 1.32892100 & 0.03931100\end{array}$

$\begin{array}{llll}-0.89508600 & 1.44939500 & 0.00003500\end{array}$ 


$\begin{array}{lrrr}\text { TS16 } & & & \\ \mathrm{C} & 1.15153300 & 0.21933100 & 0.01519800 \\ \mathrm{C} & 1.46407200 & -1.11723500 & 0.06988600 \\ \mathrm{~N} & -1.84453100 & -0.68466400 & -0.15451500 \\ \mathrm{H} & 1.43160900 & -1.69948400 & -0.84309400 \\ \mathrm{H} & 2.13559800 & 0.71250200 & -0.34088500 \\ \mathrm{H} & 1.97167700 & -1.50369700 & 0.94534700 \\ \mathrm{~N} & -2.04854800 & 0.37134900 & 0.16401500 \\ \mathrm{~N} & 0.85986300 & 1.43875800 & -0.04833900\end{array}$

\section{TS17}

$\begin{array}{lrrr}\mathrm{C} & -0.17603100 & 1.15265300 & -0.00001000 \\ \mathrm{C} & 1.04924000 & 0.44355400 & 0.00010600 \\ \mathrm{~N} & 1.00045500 & -0.86230600 & 0.00008800 \\ \mathrm{H} & 1.89482400 & -1.34714800 & 0.00016900 \\ \mathrm{H} & -0.40156800 & 2.20299400 & -0.00000100 \\ \mathrm{H} & 1.97644600 & 1.01452600 & 0.00020300 \\ \mathrm{~N} & -1.10042700 & -0.96539900 & -0.00011500 \\ \mathrm{~N} & -1.14416500 & 0.19233200 & -0.00010800\end{array}$

\section{TS18}

$\mathrm{C}$

C

$\mathrm{N}$

$\mathrm{H}$

$\mathrm{H}$

$\mathrm{H}$

$\mathrm{N}$

$\mathrm{N}$

$$
\begin{array}{rrr}
-0.53327900 & 1.26859600 & -0.19337400 \\
-1.37151500 & 0.16733200 & -0.01575200 \\
-1.33962700 & -1.11231800 & 0.04086000 \\
-2.28761200 & -1.48729200 & 0.02702900 \\
-0.41957700 & 1.86430700 & 0.72399200 \\
-2.30243200 & 0.82056300 & -0.10386100 \\
2.27759100 & -0.47223000 & -0.11403800 \\
1.41037700 & 0.18267000 & 0.15997700
\end{array}
$$

\section{TS19}

$\begin{array}{lrrc}\mathrm{C} & -0.53063000 & 1.24396400 & 0.03318500 \\ \mathrm{C} & 1.38744700 & 0.39412200 & 0.01119300 \\ \mathrm{H} & -0.78732800 & 2.28334800 & -0.10047200 \\ \mathrm{H} & 2.03577100 & 1.25187200 & 0.00944200 \\ \mathrm{~N} & -1.13282800 & 0.18551500 & 0.01225000 \\ \mathrm{~N} & -0.94452400 & -1.06376900 & -0.13692700 \\ \mathrm{H} & -1.26492900 & -1.56085100 & 0.70200000 \\ \mathrm{~N} & 1.34529200 & -0.80787300 & -0.00064300\end{array}$

\section{TS20}

$\begin{array}{lrrr}\mathrm{C} & 0.61611800 & 0.97087400 & 0.01891800 \\ \mathrm{C} & 1.09431500 & -0.33737800 & -0.00228500 \\ \mathrm{H} & 1.15001700 & 1.90963900 & 0.05742100 \\ \mathrm{H} & 2.11541500 & -0.69297900 & -0.01518700 \\ \mathrm{~N} & -0.73194500 & 0.93554600 & -0.08067000 \\ \mathrm{~N} & -1.05808500 & -0.49576300 & -0.07767900 \\ \mathrm{H} & -1.41524200 & 0.33886800 & 0.76964600 \\ \mathrm{~N} & 0.05963200 & -1.20499800 & 0.02810900\end{array}$




$\begin{array}{lrrr}\text { TS21 } & & & \\ \mathrm{C} & 2.01575900 & 0.61762200 & -0.10695200 \\ \mathrm{H} & 2.91823600 & 1.20738200 & -0.20291100 \\ \mathrm{~N} & 2.05865600 & -0.67767800 & 0.30822800 \\ \mathrm{C} & 0.03888500 & 0.00457000 & -0.18937300 \\ \mathrm{~N} & 0.80331900 & -1.12130800 & 0.35220000 \\ \mathrm{H} & 0.28363000 & -1.03893900 & -0.86386700 \\ \mathrm{~N} & 0.80059000 & 1.08277600 & -0.42963800 \\ \mathrm{~N} & -1.41644800 & 0.04556900 & -0.00465200 \\ \mathrm{O} & -2.03439100 & -0.95528100 & -0.36717100 \\ \mathrm{O} & -1.87217800 & 1.05439200 & 0.52489100\end{array}$

\section{TS22}

$\begin{array}{lrrr}\mathrm{C} & -1.84419600 & -0.80985500 & -0.04050600 \\ \mathrm{H} & -2.67437300 & -1.46819400 & 0.18802500 \\ \mathrm{~N} & -1.88542400 & 0.83668900 & 0.49326400 \\ \mathrm{C} & 0.13251300 & 0.01886500 & -0.78762700 \\ \mathrm{~N} & -0.89462200 & 1.38330400 & 0.15396100 \\ \mathrm{H} & 0.36264800 & 0.36676700 & -1.79055400 \\ \mathrm{~N} & -0.77567100 & -0.99754200 & -0.64605000 \\ \mathrm{~N} & 1.32163700 & -0.11418800 & 0.04932800 \\ \mathrm{O} & 1.21737300 & -0.76903400 & 1.08940200 \\ \mathrm{O} & 2.31017400 & 0.53022600 & -0.31217600\end{array}$

\section{TS23}

$\begin{array}{lrrr}\mathrm{C} & -1.86060400 & -0.81346000 & 0.03466900 \\ \mathrm{H} & -2.65011200 & -1.54814500 & 0.09404000 \\ \mathrm{~N} & -2.13027200 & 0.47915000 & -0.24194600 \\ \mathrm{C} & 0.03146400 & 0.08200700 & 0.03819400 \\ \mathrm{~N} & -0.90711000 & 1.02744100 & -0.25403400 \\ \mathrm{H} & -0.77680100 & 2.01323500 & -0.43928300 \\ \mathrm{~N} & -0.55193800 & -1.10570200 & 0.22039500 \\ \mathrm{~N} & 1.51462700 & 0.29074700 & -0.24385300 \\ \mathrm{O} & 2.34685500 & -0.48191700 & -0.69480300 \\ \mathrm{O} & 1.26872100 & 0.36718900 & 1.13782000\end{array}$

$\begin{array}{crrr}\text { TS24 } & & & \\ \mathrm{C} & 2.07959800 & 0.54116600 & -0.00013400 \\ \mathrm{H} & 3.05512000 & 1.00888800 & -0.00030800 \\ \mathrm{~N} & 1.95017500 & -0.82313500 & -0.00005500 \\ \mathrm{C} & 0.07155600 & 0.22377100 & 0.00023200 \\ \mathrm{~N} & 0.62526600 & -1.00417200 & 0.00021700 \\ \mathrm{H} & -0.57917300 & -1.58207600 & -0.00009800 \\ \mathrm{~N} & 0.92399500 & 1.24793500 & -0.00000200 \\ \mathrm{~N} & -1.33243700 & 0.11424400 & 0.00001600 \\ \mathrm{O} & -2.12170700 & 1.03434400 & -0.00000600 \\ \mathrm{O} & -1.69727700 & -1.12941200 & -0.00017000\end{array}$

\section{TS25}

$\begin{array}{cccc}\mathrm{C} & 1.99512200 & 0.61022000 & -0.25267700 \\ \mathrm{H} & 2.88053500 & 1.05544100 & -0.69278000 \\ \mathrm{~N} & 0.73352200 & 1.08228400 & -0.39235000 \\ \mathrm{~N} & 0.71518800 & -1.07582900 & 0.38896300 \\ \mathrm{~N} & 2.00102000 & -0.77040300 & 0.20871600 \\ \mathrm{H} & 2.31890200 & 0.29820800 & 0.92580900 \\ \mathrm{C} & 0.03419500 & 0.01410200 & -0.02313600 \\ \mathrm{~N} & -1.42652000 & 0.01303200 & -0.00520700 \\ \mathrm{O} & -1.96982000 & 0.96570200 & 0.55116500 \\ \mathrm{O} & -1.97240500 & -0.94609900 & -0.54854100\end{array}$




$\begin{array}{lrrr}\text { TS26 } & & & \\ \mathrm{C} & 1.91849800 & -1.05934500 & 0.00069300 \\ \mathrm{H} & 2.36232600 & -1.43189900 & 0.92317500 \\ \mathrm{~N} & 0.56221300 & -1.00792100 & 0.00066900 \\ \mathrm{~N} & 1.40271800 & 1.42423000 & -0.00100800 \\ \mathrm{~N} & 2.33174800 & 0.71348000 & -0.00045200 \\ \mathrm{H} & 2.36223700 & -1.43305500 & -0.92136800 \\ \mathrm{C} & -0.14787700 & 0.00023200 & -0.00004900 \\ \mathrm{~N} & -1.59991000 & 0.02735700 & -0.00001000 \\ \mathrm{O} & -2.13921700 & 0.06867400 & -1.10269900 \\ \mathrm{O} & -2.13899100 & 0.07127700 & 1.10269100\end{array}$

$\begin{array}{lrrr}\text { TS27 } & & & \\ \mathrm{C} & 1.82992600 & 0.86561500 & 0.08167800 \\ \mathrm{H} & 2.62344500 & 1.59481300 & 0.15591800 \\ \mathrm{C} & 0.01136800 & -0.14737200 & 0.05435400 \\ \mathrm{~N} & 0.53341600 & 1.07637600 & 0.29710800 \\ \mathrm{~N} & -1.54809700 & -0.25662100 & -0.28946400 \\ \mathrm{O} & -2.40690500 & 0.53054700 & -0.62378700 \\ \mathrm{O} & -1.31300300 & -0.51162000 & 1.03005600 \\ \mathrm{~N} & 0.87170100 & -1.12019500 & -0.28972900 \\ \mathrm{~N} & 2.02828800 & -0.43484100 & -0.25147700 \\ \mathrm{H} & 2.89088600 & -0.90871500 & -0.48732400\end{array}$

$\begin{array}{lrrr}\text { TS28 } & & & \\ \mathrm{C} & -2.06899900 & 0.50861300 & -0.18760000 \\ \mathrm{H} & -2.95578500 & 0.97368100 & -0.60046100 \\ \mathrm{H} & -1.50828400 & 1.35677400 & 0.55792800 \\ \mathrm{~N} & -0.72001000 & -1.09978100 & 0.35630500 \\ \mathrm{~N} & -2.01658700 & -0.76348000 & 0.28631400 \\ \mathrm{C} & -0.03320800 & -0.02298100 & -0.05825000 \\ \mathrm{~N} & -0.74526000 & 1.03717300 & -0.46167000 \\ \mathrm{~N} & 1.42430500 & 0.01428500 & -0.00539500 \\ \mathrm{O} & 2.01118900 & -0.94673900 & -0.49585300 \\ \mathrm{O} & 1.92383200 & 1.00153500 & 0.53194700\end{array}$

\section{TS29}

$\begin{array}{cccc}\mathrm{C} & -1.91860800 & -1.05919500 & 0.00049300 \\ \mathrm{H} & -2.36238300 & -1.43181300 & 0.92297800 \\ \mathrm{H} & -2.36241300 & -1.43278800 & -0.92158200 \\ \mathrm{~N} & -1.40247300 & 1.42421600 & -0.00067400 \\ \mathrm{~N} & -2.33159200 & 0.71355700 & -0.00034600 \\ \mathrm{C} & 0.14782300 & 0.00015900 & -0.00002700 \\ \mathrm{~N} & -0.56226400 & -1.00800500 & 0.00045800 \\ \mathrm{~N} & 1.59985200 & 0.02729000 & -0.00000600 \\ \mathrm{O} & 2.13899700 & 0.07086700 & 1.10268300 \\ \mathrm{O} & 2.13910800 & 0.06906100 & -1.10271000\end{array}$

$\begin{array}{lrrr}\text { TS30 } & & & \\ \mathrm{C} & 2.08394700 & 0.35109600 & -0.21611100 \\ \mathrm{H} & 3.04773200 & 0.81202000 & -0.37993200 \\ \mathrm{H} & 0.74328300 & 2.02210600 & -0.41230200 \\ \mathrm{~N} & 0.52796200 & -1.11267400 & 0.21517500 \\ \mathrm{~N} & 1.87734300 & -0.93068700 & 0.04779100 \\ \mathrm{C} & -0.04581500 & 0.09396200 & 0.04836500 \\ \mathrm{~N} & 0.89789800 & 1.03261600 & -0.27595000 \\ \mathrm{~N} & -1.49795300 & 0.28391300 & -0.24984800 \\ \mathrm{O} & -2.35605900 & -0.43987300 & -0.70042100 \\ \mathrm{O} & -1.22601100 & 0.38779200 & 1.15523700\end{array}$




$\begin{array}{lrrr}\text { TS31 } & & & \\ \mathrm{C} & 1.94577200 & 0.67978900 & -0.00012100 \\ \mathrm{H} & 2.76027000 & 1.38975300 & -0.00019400 \\ \mathrm{H} & -0.55038900 & 1.57975300 & -0.00008400 \\ \mathrm{~N} & 0.90022500 & -1.25196000 & 0.00007800 \\ \mathrm{~N} & 2.12516900 & -0.66521600 & 0.00001300 \\ \mathrm{C} & 0.06997200 & -0.20675300 & -0.00005000 \\ \mathrm{~N} & 0.63739400 & 1.01478600 & -0.00018200 \\ \mathrm{~N} & -1.33907900 & -0.09089200 & 0.00005900 \\ \mathrm{O} & -2.12902600 & -1.00954300 & 0.00012500 \\ \mathrm{O} & -1.69226300 & 1.15269900 & 0.00006700\end{array}$

\section{TS32}

$\begin{array}{lrrr}\mathrm{C} & 0.90238300 & 1.10370600 & 0.23728000 \\ \mathrm{C} & 0.01849900 & 0.01635900 & 0.16610800 \\ \mathrm{~N} & 0.81040100 & -1.14292600 & -0.20149800 \\ \mathrm{H} & 0.31516400 & -0.93257900 & 0.97163400 \\ \mathrm{H} & 0.69303800 & 2.13941300 & 0.46081500 \\ \mathrm{~N} & 2.07195200 & -0.69483600 & -0.20417500 \\ \mathrm{~N} & 2.13633400 & 0.63784900 & 0.01231800 \\ \mathrm{~N} & -1.43536500 & 0.01518700 & -0.00246800 \\ \mathrm{O} & -2.01874500 & -1.04834100 & 0.18340600 \\ \mathrm{O} & -1.93334900 & 1.09407300 & -0.31865800\end{array}$

\section{TS33}

$\begin{array}{lrrc}\mathrm{C} & -0.93504800 & -1.01589800 & 0.49513500 \\ \mathrm{C} & 0.08098100 & 0.03184700 & 0.71955000 \\ \mathrm{~N} & -0.78688600 & 1.40691400 & -0.09442500 \\ \mathrm{H} & 0.27827600 & 0.34752600 & 1.74509200 \\ \mathrm{H} & -0.61461400 & -1.97697000 & 0.92813100 \\ \mathrm{~N} & -1.82036700 & 1.10777200 & -0.51504500 \\ \mathrm{~N} & -2.07555200 & -0.99432900 & -0.04781200 \\ \mathrm{~N} & 1.34090600 & -0.12366700 & -0.02126500 \\ \mathrm{O} & 2.24004900 & 0.66105500 & 0.29448300 \\ \mathrm{O} & 1.36670500 & -0.94144000 & -0.94592100\end{array}$

\section{TS34}

$\begin{array}{lrrr}\mathrm{C} & -1.02312900 & 1.10395700 & -0.27145900 \\ \mathrm{C} & 0.04769700 & 0.23341200 & 0.04116700 \\ \mathrm{~N} & -0.57006800 & -0.96790100 & 0.22637600 \\ \mathrm{H} & -0.14186500 & -1.86709600 & 0.41572900 \\ \mathrm{H} & -0.98194500 & 2.15914600 & -0.49588600 \\ \mathrm{~N} & -1.91125500 & -0.88328300 & 0.01989600 \\ \mathrm{~N} & -2.16712100 & 0.39949600 & -0.24600000 \\ \mathrm{~N} & 1.53203200 & 0.31592600 & -0.26796900 \\ \mathrm{O} & 2.32796700 & -0.47029400 & -0.71359000 \\ \mathrm{O} & 1.27094300 & 0.42455200 & 1.13056400\end{array}$

$\begin{array}{lrrr}\text { TS35 } & & & \\ \mathrm{C} & -1.06298500 & 1.19680000 & 0.00268200 \\ \mathrm{C} & -0.04570700 & 0.24027600 & -0.02580000 \\ \mathrm{~N} & -0.60646900 & -0.98248700 & -0.02188000 \\ \mathrm{H} & 0.59433500 & -1.57400200 & 0.00955700 \\ \mathrm{H} & -1.02813400 & 2.27562800 & 0.00679700 \\ \mathrm{~N} & -1.93133200 & -0.83536400 & 0.00619400 \\ \mathrm{~N} & -2.21279400 & 0.49467800 & 0.01468900 \\ \mathrm{~N} & 1.35152000 & 0.11153000 & -0.00194800 \\ \mathrm{O} & 1.71336700 & -1.13269100 & 0.01695400 \\ \mathrm{O} & 2.14656800 & 1.02736800 & 0.00091800\end{array}$




$\begin{array}{lrrr}\text { TS36 } & & & \\ \mathrm{C} & 0.80431600 & 1.07352500 & 0.03707900 \\ \mathrm{C} & 0.02924200 & -0.09990600 & -0.01712200 \\ \mathrm{~N} & 0.63403000 & -1.25352800 & -0.06547900 \\ \mathrm{H} & 0.04589900 & -2.08567500 & -0.05175200 \\ \mathrm{H} & 0.53194600 & 2.11189100 & 0.06958100 \\ \mathrm{~N} & 2.49602500 & -0.46534000 & -0.00105700 \\ \mathrm{~N} & 2.09038300 & 0.62540900 & 0.03024100 \\ \mathrm{~N} & -1.44518300 & 0.04166600 & 0.00064400 \\ \mathrm{O} & -2.10139900 & -0.99725800 & 0.07719800 \\ \mathrm{O} & -1.89934900 & 1.18408600 & -0.06319900\end{array}$

$\begin{array}{lrrr}\text { TS37 } & & & \\ \mathrm{C} & 0.41848100 & -1.03351100 & -0.10652000 \\ \mathrm{C} & -0.18820000 & 0.29562100 & 0.07209900 \\ \mathrm{~N} & 0.23199000 & 1.48937500 & 0.12977700 \\ \mathrm{H} & -0.56860700 & 2.12874800 & 0.07987600 \\ \mathrm{H} & 0.59437000 & -1.56652900 & 0.83412700 \\ \mathrm{~N} & 3.55979200 & 0.08985500 & -0.20459400 \\ \mathrm{~N} & 2.54113800 & -0.24076500 & 0.12575400 \\ \mathrm{~N} & -1.64421900 & -0.08713700 & -0.03105900 \\ \mathrm{O} & -2.55283800 & 0.71836100 & -0.11040000 \\ \mathrm{O} & -1.72570700 & -1.33013200 & 0.00457100\end{array}$

$\begin{array}{lrrr}\text { TS38 } & & & \\ \mathrm{C} & -0.84654000 & 1.09795300 & -0.08382900 \\ \mathrm{C} & -0.01400300 & -0.02209500 & -0.01984100 \\ \mathrm{H} & -0.66227800 & 2.12679300 & -0.36108300 \\ \mathrm{~N} & -2.02308000 & -0.79645100 & 0.04464300 \\ \mathrm{~N} & 1.44606700 & -0.00691600 & 0.00260900 \\ \mathrm{O} & 2.03218000 & -1.08116300 & -0.05604600 \\ \mathrm{O} & 1.95527700 & 1.11376000 & 0.07336100 \\ \mathrm{~N} & -0.72627900 & -1.14597200 & 0.03228200 \\ \mathrm{~N} & -2.18696100 & 0.52691100 & -0.08911100 \\ \mathrm{H} & -1.64234400 & 1.11427200 & 0.91162500\end{array}$

TS39

$\begin{array}{lrrr}\mathrm{C} & -0.92993000 & -1.18065200 & 0.32291900 \\ \mathrm{C} & 0.14401100 & 0.52041800 & -0.13262400 \\ \mathrm{H} & -0.73514400 & -1.49402500 & 1.34775400 \\ \mathrm{~N} & -2.66024000 & 0.46934400 & -0.05979600 \\ \mathrm{~N} & 1.49919100 & 0.00458400 & -0.02463800 \\ \mathrm{O} & 2.21846000 & 0.51909600 & 0.82676900 \\ \mathrm{O} & 1.79832700 & -0.89956400 & -0.80207200 \\ \mathrm{~N} & -0.44427100 & 1.53025300 & -0.42032900 \\ \mathrm{~N} & -2.09731600 & -0.51610200 & 0.18676700 \\ \mathrm{H} & -0.76519900 & -1.91738500 & -0.46113300\end{array}$

$\begin{array}{lrrr}\text { TS40 } & & & \\ \mathrm{C} & -0.99807800 & 1.07531400 & -0.30521500 \\ \mathrm{C} & 0.01547200 & 0.18009900 & 0.05251400 \\ \mathrm{H} & -1.00575800 & 2.11841100 & -0.57554400 \\ \mathrm{~N} & -1.81807200 & -0.98249100 & 0.12117300 \\ \mathrm{~N} & 1.56408000 & 0.24546500 & -0.30983500 \\ \mathrm{O} & 2.41581400 & -0.55000400 & -0.60338300 \\ \mathrm{O} & 1.32424800 & 0.58580600 & 1.01111900 \\ \mathrm{~N} & -2.09805200 & 0.28687100 & -0.22583500 \\ \mathrm{H} & -3.06375100 & 0.54014500 & -0.39731300 \\ \mathrm{~N} & -0.49871900 & -1.04662500 & 0.30409400\end{array}$




$\begin{array}{lrrr}\text { TS41 } & & & \\ \mathrm{C} & -0.66268100 & 1.10080100 & 0.00027100 \\ \mathrm{C} & -0.05051900 & -0.15182200 & 0.00004200 \\ \mathrm{H} & 0.73702900 & 1.64546300 & 0.00001900 \\ \mathrm{~N} & -2.08783100 & -0.70149100 & -0.00018100 \\ \mathrm{~N} & 1.35473500 & -0.09468300 & -0.00001100 \\ \mathrm{O} & 2.11551000 & -1.03139100 & 0.00035000 \\ \mathrm{O} & 1.77957100 & 1.14035300 & -0.00038700 \\ \mathrm{~N} & -0.87642500 & -1.22320000 & -0.00028200 \\ \mathrm{~N} & -1.93767600 & 0.67167600 & 0.00019900 \\ \mathrm{H} & -2.78809900 & 1.22284300 & 0.00032400\end{array}$

\section{TS42}

$\begin{array}{lrrr}\mathrm{C} & -0.90280600 & 1.10771600 & 0.00015500 \\ \mathrm{C} & 0.01185100 & 0.02041500 & -0.00000600 \\ \mathrm{H} & -0.50681400 & 2.12078500 & 0.00027400 \\ \mathrm{~N} & -1.86398300 & -1.27281200 & -0.00012800 \\ \mathrm{~N} & 1.43120900 & 0.02693400 & -0.00002200 \\ \mathrm{O} & 2.01536000 & -1.05813600 & -0.00016700 \\ \mathrm{O} & 1.96495000 & 1.14116700 & 0.00010600 \\ \mathrm{~N} & -0.71309800 & -1.12772900 & -0.00011800 \\ \mathrm{~N} & -2.16131300 & 0.78776700 & 0.00013700 \\ \mathrm{H} & -2.83963800 & 1.54706800 & 0.00023600\end{array}$

$\begin{array}{lrrr}\text { TS43 } & & & \\ \mathrm{C} & 0.00540100 & 1.56576900 & -0.04822500 \\ \mathrm{C} & 0.30338900 & 0.27897000 & -0.71615700 \\ \mathrm{H} & 0.88222700 & 2.22646300 & -0.11104300 \\ \mathrm{~N} & -2.56596500 & -1.29335000 & -0.20746100 \\ \mathrm{~N} & 1.22245100 & -0.50337000 & 0.01638100 \\ \mathrm{O} & 1.06087900 & -1.49999500 & 0.69285500 \\ \mathrm{O} & 2.29201300 & 0.05914700 & -0.29931500 \\ \mathrm{~N} & -1.58494100 & -0.75704100 & -0.12605500 \\ \mathrm{~N} & -1.13843400 & 1.89266300 & 0.43013300 \\ \mathrm{H} & -1.08987700 & 2.85958300 & 0.75803300\end{array}$

\section{TS44}

$\begin{array}{lrrr}\mathrm{C} & 0.84776300 & 1.11655100 & 0.07834000 \\ \mathrm{C} & 0.01042500 & 0.00711900 & -0.02158600 \\ \mathrm{H} & 0.58691800 & 2.16272800 & 0.14797800 \\ \mathrm{~N} & -1.44521100 & -0.00631100 & 0.00190100 \\ \mathrm{O} & -2.00024600 & -1.09063500 & 0.15859900 \\ \mathrm{O} & -1.98956800 & 1.09093700 & -0.12835100 \\ \mathrm{~N} & 0.72628100 & -1.11247300 & -0.19968000 \\ \mathrm{~N} & 2.12143200 & 0.68356400 & 0.11435800 \\ \mathrm{~N} & 2.11269000 & -0.62820500 & -0.11231300 \\ \mathrm{H} & 1.57610800 & -1.46320300 & 0.63965300\end{array}$

$\begin{array}{lrrr}\text { TS45 } & & & \\ \mathrm{C} & 0.83403800 & 1.11464600 & -0.09960900 \\ \mathrm{C} & 0.01536300 & -0.00378500 & 0.01341000 \\ \mathrm{H} & 0.58210400 & 2.16209700 & -0.17006400 \\ \mathrm{~N} & -1.44476500 & -0.00793600 & 0.00048400 \\ \mathrm{O} & -2.01210100 & -1.07991100 & -0.18230600 \\ \mathrm{O} & -1.97593600 & 1.09069500 & 0.17375400 \\ \mathrm{~N} & 0.72032300 & -1.13389400 & 0.16012900 \\ \mathrm{~N} & 2.10348400 & 0.65697700 & -0.16514400 \\ \mathrm{~N} & 1.99482100 & -0.78756400 & 0.00388500 \\ \mathrm{H} & 2.60874200 & -0.00661300 & 0.76020500\end{array}$




\section{TS46}

$\begin{array}{lrrr}\mathrm{C} & -0.63699600 & 1.07916600 & 0.23783500 \\ \mathrm{C} & 0.03339800 & -0.15143100 & 0.06401900 \\ \mathrm{H} & -0.23866300 & 2.05797000 & 0.45860400 \\ \mathrm{~N} & 1.55429200 & -0.30263100 & -0.27751700 \\ \mathrm{O} & 2.39748200 & 0.46417600 & -0.67524400 \\ \mathrm{O} & 1.29719100 & -0.43721800 & 1.09085200 \\ \mathrm{~N} & -0.85957700 & -1.12147400 & -0.25682900 \\ \mathrm{~N} & -1.95232100 & 0.86725000 & 0.03108900 \\ \mathrm{~N} & -2.00194100 & -0.43540900 & -0.23292300 \\ \mathrm{H} & -2.88030900 & -0.89419200 & -0.44133400\end{array}$




\section{Intermediates:}

$\begin{array}{lccc}\text { IM1 } & & & \\ \text { C } & -0.79621700 & 0.76931600 & 0.00022800 \\ \text { H } & -1.80973000 & -1.09660000 & 0.00066800 \\ \text { H } & -1.60766200 & 1.48059300 & -0.00106900 \\ \text { N } & 0.47797900 & 1.09453100 & 0.00014700 \\ \text { C } & 0.28707300 & -1.23024600 & 0.00009800 \\ \text { N } & 1.08228500 & -0.13587300 & -0.00026600 \\ \text { H } & 2.09110100 & -0.16120100 & 0.00041700 \\ \text { N } & -0.93438500 & -0.59540200 & -0.00016200\end{array}$

$\begin{array}{lrrc}\text { IM2 } & & & \\ \text { C } & 1.08980600 & -0.31325500 & -0.00056300 \\ \text { H } & -1.65061400 & -0.60362400 & 0.89125200 \\ \text { H } & 2.15427100 & -0.51489400 & 0.00023300 \\ \text { N } & 0.61816500 & 1.05580100 & 0.00010500 \\ \text { C } & -1.04479700 & -0.40552200 & -0.00035600 \\ \text { N } & -0.65209900 & 1.00920900 & 0.00017600 \\ \text { H } & -1.65016100 & -0.60423800 & -0.89189800 \\ \text { N } & 0.15914100 & -1.20280700 & 0.00056600\end{array}$

\section{IM3}

$\begin{array}{lrrr}\mathrm{C} & 1.42342100 & -0.98510600 & 0.13400200 \\ \mathrm{H} & -0.16210300 & 1.81142900 & 0.62608700 \\ \mathrm{H} & 2.49468200 & -1.16437900 & -0.06943200 \\ \mathrm{H} & 1.96787900 & 0.93819900 & -0.39401500 \\ \mathrm{~N} & -1.06713000 & 0.09881400 & 0.01309000 \\ \mathrm{~N} & -1.96932700 & -0.59192200 & -0.05214000 \\ \mathrm{C} & -0.03963100 & 0.94969900 & -0.01849700 \\ \mathrm{~N} & 1.23599900 & 0.29699400 & -0.08318900\end{array}$

\section{IM4}

C

\section{IM5}

$\begin{array}{lrrr}\mathrm{C} & -1.93814500 & 0.47059900 & -0.09550300 \\ \mathrm{H} & 2.05452300 & -1.05893400 & -0.03924400 \\ \mathrm{H} & -2.75393200 & 1.03761300 & 0.29975100 \\ \mathrm{H} & 2.33834400 & 1.17992800 & -0.02316500 \\ \mathrm{~N} & 0.00079400 & -1.00769600 & 0.01337300 \\ \mathrm{~N} & -0.97808700 & -0.20724600 & 0.02954400 \\ \mathrm{C} & 1.23243500 & -0.34136700 & -0.00631700 \\ \mathrm{~N} & 1.34805400 & 0.93865700 & 0.01045100\end{array}$

$$
\begin{array}{rrr}
2.11215900 & -0.07496400 & -0.48382400 \\
0.11352200 & 1.62957000 & -0.27330900 \\
2.83976500 & -0.83393800 & -0.13559500 \\
1.04159000 & -0.84957400 & 1.10684000 \\
-1.20034300 & 0.08760600 & -0.05040900 \\
-2.21918500 & -0.41402100 & -0.16880100 \\
-0.04485400 & 0.69540700 & 0.24931600 \\
1.07685600 & -0.19768700 & 0.32051200
\end{array}
$$

$\begin{array}{llll}1.34805400 & 0.93865700 & 0.01045100\end{array}$ 


$\begin{array}{lrrr}\text { IM6 } & & & \\ \text { C } & 1.08948300 & -0.31399700 & 0.00029900 \\ \text { H } & -1.65055100 & -0.60211100 & 0.89196500 \\ \text { H } & 2.15380100 & -0.51644500 & -0.00032500 \\ \text { H } & -1.65136300 & -0.60298000 & -0.89079800 \\ \text { N } & -0.65120000 & 1.00964300 & -0.00032100 \\ \text { N } & 0.61898300 & 1.05522800 & 0.00006400 \\ \text { C } & -1.04512000 & -0.40468900 & 0.00023100 \\ \text { N } & 0.15820800 & -1.20292100 & -0.00031800\end{array}$

$\begin{array}{lrrr}\text { IM7 } & & & \\ \text { C } & -1.04174700 & 0.44647900 & 0.00031100 \\ \text { C } & 0.28428900 & 1.11709900 & -0.00029700 \\ \text { N } & 1.19546900 & -0.03031600 & -0.00020800 \\ \text { H } & 0.47607500 & 1.72922100 & -0.88834500 \\ \mathrm{H} & -2.01371500 & 0.92568900 & 0.00013500 \\ \mathrm{H} & 0.47545200 & 1.72556900 & 0.89061000 \\ \mathrm{~N} & 0.53012700 & -1.09679600 & 0.00029400 \\ \mathrm{~N} & -0.92460500 & -0.83888000 & -0.00044000\end{array}$

$\begin{array}{lrrr}\text { IM8 } & & & \\ \text { C } & -1.16250700 & 0.25769000 & 0.00001600 \\ \text { C } & -1.16124400 & -1.18238700 & -0.00003900 \\ \text { N } & 1.71856900 & -0.68739900 & -0.00000400 \\ \text { H } & -1.54305600 & -1.62612200 & 0.92103100 \\ \text { H } & -2.26559300 & 0.44819400 & 0.00003200 \\ \text { H } & -1.54386300 & -1.62601600 & -0.92082500 \\ \text { N } & 1.79045500 & 0.43432300 & -0.00002900 \\ \text { N } & -0.75259300 & 1.44623700 & 0.00001800\end{array}$

\section{IM9}

$\begin{array}{lrrc}\mathrm{C} & -0.06474400 & 0.96097700 & -0.00000200 \\ \mathrm{C} & 1.24961500 & 0.37028300 & 0.00012400 \\ \mathrm{~N} & 1.42619900 & -0.91134400 & 0.00013400 \\ \mathrm{H} & 2.42534800 & -1.11533900 & 0.00022900 \\ \mathrm{H} & -0.30726700 & 2.01250100 & -0.00001900 \\ \mathrm{H} & 2.06416000 & 1.10350600 & 0.00020600 \\ \mathrm{~N} & -1.94438100 & -0.63730800 & -0.00019200 \\ \mathrm{~N} & -1.09488500 & 0.12176200 & -0.00010600\end{array}$

\section{IM10}

$\begin{array}{lrrr}\mathrm{C} & -1.86226100 & 0.65497500 & 0.05575600 \\ \mathrm{H} & -2.72721300 & 1.28467100 & -0.11553900 \\ \mathrm{~N} & -1.83990700 & -0.71180200 & -0.47509700 \\ \mathrm{C} & 0.00422300 & -0.19271400 & 0.64297000 \\ \mathrm{~N} & -0.73126700 & -1.21929500 & -0.14794500 \\ \mathrm{H} & 0.23711100 & -0.58577600 & 1.63463400 \\ \mathrm{~N} & -0.80192000 & 0.99476300 & 0.69287800 \\ \mathrm{~N} & 1.29726800 & 0.07442100 & -0.08089900 \\ \mathrm{O} & 1.19014900 & 0.60311600 & -1.18165600 \\ \mathrm{O} & 2.33099000 & -0.28300000 & 0.47740400\end{array}$




$\begin{array}{lccc}\text { IM11 } & & & \\ \text { C } & 1.99196900 & 0.90776200 & -0.04061600 \\ \text { H } & 2.68500900 & 1.73461200 & -0.09914800 \\ \text { N } & 2.38801300 & -0.34660400 & -0.28754200 \\ \text { C } & 0.22938200 & -0.19417400 & 0.24259400 \\ \text { N } & 1.23561200 & -1.03069100 & -0.12746700 \\ \text { H } & 1.21085400 & -2.03704000 & -0.21301100 \\ \text { N } & 0.67877700 & 1.05464600 & 0.29723400 \\ \text { N } & -2.05132200 & 0.30042300 & -0.45571600 \\ \text { O } & -3.12101600 & 0.15285500 & -0.10313900 \\ \text { O } & -1.00167500 & -0.63079500 & 0.49248100\end{array}$

$\begin{array}{lrrr}\text { IM12 } & & & \\ \text { C } & 2.07387800 & -0.56894100 & 0.00004300 \\ \text { H } & 3.02863200 & -1.07914900 & 0.00008700 \\ \text { N } & 1.99677300 & 0.81611900 & 0.00000300 \\ \text { C } & 0.08787000 & -0.12375200 & -0.00005800 \\ \text { N } & 0.70077200 & 1.09949300 & -0.00004900 \\ \text { H } & -1.12418600 & 1.70137200 & 0.00009900 \\ \text { N } & 0.89834900 & -1.20324000 & 0.00000200 \\ \text { N } & -1.28160900 & -0.14983900 & -0.00001300 \\ \text { O } & -1.99700100 & -1.12325000 & -0.00000300 \\ \text { O } & -1.88736500 & 1.07277500 & 0.00004200\end{array}$

$\begin{array}{lcrc}\text { IM13 } & & & \\ \text { C } & 2.05837900 & -0.57067300 & 0.24145400 \\ \text { H } & 2.60053300 & -0.51435700 & 1.19235000 \\ \text { N } & 0.71101100 & -1.06306600 & 0.43586200 \\ \text { N } & 0.74384000 & 1.07450400 & -0.46826400 \\ \text { N } & 1.97171600 & 0.77874200 & -0.33722100 \\ \text { H } & 2.61284100 & -1.21705100 & -0.44891500 \\ \text { C } & 0.02104800 & -0.07425700 & 0.01362800 \\ \text { N } & -1.43587300 & -0.01906100 & 0.01120700 \\ \text { O } & -2.02982100 & -0.84705000 & -0.67607200 \\ \text { O } & -1.92327700 & 0.87244400 & 0.70544500\end{array}$

\section{IM14}

$\begin{array}{cccc}\mathrm{C} & 2.01124200 & 0.86347300 & -0.08760600 \\ \mathrm{H} & 2.79161100 & 1.60023700 & -0.21497300 \\ \mathrm{C} & 0.22011400 & -0.16417500 & 0.28000100 \\ \mathrm{~N} & 0.75349800 & 1.08883800 & 0.27422400 \\ \mathrm{~N} & -2.00747200 & 0.20842900 & -0.49655500 \\ \mathrm{O} & -3.10417500 & 0.09502400 & -0.18184600 \\ \mathrm{O} & -1.05177500 & -0.41819000 & 0.62533600 \\ \mathrm{~N} & 1.06028100 & -1.15280100 & -0.06349800 \\ \mathrm{~N} & 2.19784300 & -0.46584400 & -0.27800200 \\ \mathrm{H} & 3.03881900 & -0.96104500 & -0.54049600\end{array}$

\section{IM15}

$\begin{array}{lccc}\mathrm{C} & 2.05840100 & 0.57037200 & 0.24107900 \\ \mathrm{H} & 2.60093000 & 0.51483300 & 1.19184400 \\ \mathrm{H} & 2.61251600 & 1.21661500 & -0.44972100 \\ \mathrm{~N} & 0.74346600 & -1.07438100 & -0.46832500 \\ \mathrm{~N} & 1.97135200 & -0.77852400 & -0.33688700 \\ \mathrm{C} & 0.02109800 & 0.07461000 & 0.01293100 \\ \mathrm{~N} & 0.71126500 & 1.06292700 & 0.43615600 \\ \mathrm{~N} & -1.43577200 & 0.01903900 & 0.01112100 \\ \mathrm{O} & -1.92249400 & -0.87308800 & 0.70505800 \\ \mathrm{O} & -2.03033300 & 0.84749200 & -0.67513800\end{array}$




$\begin{array}{lccc}\text { IM16 } & & & \\ \text { C } & 2.33269000 & 0.17412800 & -0.26481200 \\ \text { H } & 3.33486100 & 0.48047800 & -0.52699600 \\ \text { H } & 1.26376000 & 2.03907800 & -0.23754900 \\ \text { N } & 0.61034500 & -1.02589300 & 0.31786600 \\ \text { N } & 1.94749000 & -1.06761100 & -0.01815100 \\ \text { C } & 0.22889400 & 0.24142700 & 0.25034800 \\ \text { N } & 1.28049700 & 1.03538800 & -0.12846900 \\ \text { N } & -2.02662100 & -0.25800300 & -0.48118400 \\ \text { O } & -3.09194400 & -0.19044800 & -0.09651500 \\ \text { O } & -0.98931900 & 0.71544100 & 0.47412600\end{array}$

$\begin{array}{lrrr}\text { IM17 } & & & \\ \text { C } & -1.98212100 & 0.68223700 & 0.00013200 \\ \mathrm{H} & -2.84108500 & 1.34024000 & 0.00021700 \\ \mathrm{H} & 1.13099400 & 1.71816300 & 0.00006600 \\ \mathrm{~N} & -0.88385000 & -1.20424200 & -0.00005500 \\ \mathrm{~N} & -2.11292400 & -0.69541200 & 0.00003400 \\ \mathrm{C} & -0.08669100 & -0.09776000 & 0.00000900 \\ \mathrm{~N} & -0.71685600 & 1.10446200 & 0.00012100 \\ \mathrm{~N} & 1.28413100 & -0.12999700 & -0.00006500 \\ \mathrm{O} & 1.99826200 & -1.10311600 & -0.00015600 \\ \mathrm{O} & 1.89291900 & 1.09199700 & -0.00001500\end{array}$

$\begin{array}{lrrr}\text { IM18 } & & & \\ \mathrm{C} & 0.87263400 & 1.15928200 & 0.05953400 \\ \mathrm{C} & -0.02470300 & 0.12181800 & 0.65389700 \\ \mathrm{~N} & 0.76938500 & -1.10543900 & 0.46937500 \\ \mathrm{H} & -0.25178700 & 0.25691600 & 1.71392200 \\ \mathrm{H} & 0.65442000 & 2.21776600 & -0.02296400 \\ \mathrm{~N} & 1.86923600 & -0.81569900 & -0.04973400 \\ \mathrm{~N} & 1.98274300 & 0.64794600 & -0.33892400 \\ \mathrm{~N} & -1.31942300 & -0.02063100 & -0.09125600 \\ \mathrm{O} & -2.35523200 & 0.14790600 & 0.54857000 \\ \mathrm{O} & -1.22024400 & -0.28597100 & -1.28579300\end{array}$

\section{IM19}

$\begin{array}{lrrr}\mathrm{C} & -0.96262900 & 1.10284900 & 0.21406100 \\ \mathrm{C} & -0.21661000 & -0.06322600 & 0.34179700 \\ \mathrm{~N} & -1.09721900 & -1.03659700 & -0.03460900 \\ \mathrm{H} & -0.95134500 & -2.03821200 & -0.03575900 \\ \mathrm{H} & -0.66216100 & 2.12129000 & 0.40995500 \\ \mathrm{~N} & -2.30616000 & -0.53389100 & -0.33990300 \\ \mathrm{~N} & -2.21736200 & 0.78679200 & -0.20081000 \\ \mathrm{~N} & 2.06878700 & 0.19776200 & -0.52113800 \\ \mathrm{O} & 3.15336300 & 0.04916100 & -0.20899400 \\ \mathrm{O} & 1.04071300 & -0.32657100 & 0.70472900\end{array}$

$\begin{array}{lrrr}\text { IM20 } & & & \\ \text { C } & -1.03621400 & 1.15573400 & -0.00044900 \\ \text { C } & -0.06461400 & 0.12587100 & 0.00021000 \\ \text { N } & -0.68740200 & -1.08450400 & 0.00056000 \\ \text { H } & 1.15477700 & -1.70673300 & -0.00070400 \\ \text { H } & -0.91606600 & 2.22974900 & -0.00130900 \\ \text { N } & -1.97939000 & -0.83015700 & 0.00036500 \\ \text { N } & -2.21162800 & 0.54362900 & -0.00034700 \\ \text { N } & 1.29972600 & 0.14488500 & 0.00001900 \\ \text { O } & 1.91237000 & -1.07262200 & -0.00075100 \\ \text { O } & 2.01476900 & 1.11892000 & 0.00065900\end{array}$




$\begin{array}{lrrr}\text { IM21 } & & & \\ \text { C } & -0.88163500 & -0.71088600 & -0.00015900 \\ \text { C } & 0.08747800 & 0.34123100 & 0.00006400 \\ \text { N } & -0.14692300 & 1.59435400 & 0.00026400 \\ \text { H } & 0.73860600 & 2.10711100 & 0.00038300 \\ \text { H } & -0.70141400 & -1.77306600 & -0.00031000 \\ \text { N } & -3.22680900 & 0.05502600 & -0.00017800 \\ \text { N } & -2.14975200 & -0.31021200 & -0.00017100 \\ \text { N } & 1.52314100 & -0.19502100 & 0.00004100 \\ \text { O } & 2.41404100 & 0.64933500 & 0.00022000 \\ \text { O } & 1.67722700 & -1.41497800 & -0.00011900\end{array}$

\section{IM22}

$\begin{array}{lrrr}\mathrm{C} & -0.89790400 & 1.08187000 & -0.31210200 \\ \mathrm{C} & -0.02148300 & -0.07407600 & -0.00532300 \\ \mathrm{H} & -0.79535300 & 1.46238100 & -1.33441100 \\ \mathrm{~N} & -2.08150200 & -0.73804000 & 0.20535500 \\ \mathrm{~N} & 1.43713300 & -0.01029600 & 0.00194400 \\ \mathrm{O} & 2.06015100 & -0.97098500 & -0.43899700 \\ \mathrm{O} & 1.90402700 & 1.03517700 & 0.46271800 \\ \mathrm{~N} & -0.66274400 & -1.13833100 & 0.31284300 \\ \mathrm{~N} & -2.21094700 & 0.46716400 & -0.14016900 \\ \mathrm{H} & -0.77532900 & 1.91384300 & 0.38937400\end{array}$

$\begin{array}{lrrr}\text { IM23 } & & & \\ \mathrm{C} & -1.26150500 & 1.10244500 & -0.11819700 \\ \mathrm{C} & -0.21028400 & 0.26258300 & 0.23917000 \\ \mathrm{H} & -1.33765900 & 2.16875200 & -0.25722300 \\ \mathrm{~N} & -1.94128100 & -1.04055500 & -0.02163800 \\ \mathrm{~N} & 2.03566400 & -0.24553200 & -0.47099000 \\ \mathrm{O} & 3.12104300 & -0.14074900 & -0.12975000 \\ \mathrm{O} & 1.05644200 & 0.60140100 & 0.52790800 \\ \mathrm{~N} & -2.29888900 & 0.24012800 & -0.24784100 \\ \mathrm{H} & -3.26194800 & 0.43898300 & -0.48490600 \\ \mathrm{~N} & -0.65114300 & -1.02305900 & 0.28775700\end{array}$

\section{IM24}

$\begin{array}{lrrr}\mathrm{C} & -0.73767100 & 1.17579300 & 0.00001100 \\ \mathrm{C} & -0.05780100 & -0.06954200 & -0.00000800 \\ \mathrm{H} & 1.13194000 & 1.71527900 & 0.00005800 \\ \mathrm{~N} & -2.07105600 & -0.73017200 & 0.00000200 \\ \mathrm{~N} & 1.31831000 & -0.11718000 & -0.00001200 \\ \mathrm{O} & 2.02417800 & -1.09100000 & 0.00002400 \\ \mathrm{O} & 1.91946300 & 1.09855500 & -0.00001700 \\ \mathrm{~N} & -0.85064800 & -1.18605800 & -0.00001700 \\ \mathrm{~N} & -1.97610800 & 0.66378800 & 0.00000500 \\ \mathrm{H} & -2.85173600 & 1.17413100 & 0.00001100\end{array}$

$\begin{array}{lrrr}\text { IM25 } & & & \\ \mathrm{C} & -1.10465500 & -1.15846600 & 0.01300800 \\ \mathrm{C} & -0.13183700 & -0.08556600 & 0.11144800 \\ \mathrm{H} & -0.66385800 & -2.15800100 & 0.00993400 \\ \mathrm{~N} & -0.99879700 & 2.22264600 & -0.01862300 \\ \mathrm{~N} & 1.28491200 & -0.23023200 & 0.00350300 \\ \mathrm{O} & 1.96435500 & 0.80152800 & -0.04441800 \\ \mathrm{O} & 1.71819900 & -1.38442500 & -0.01074800 \\ \mathrm{~N} & -0.56592900 & 1.17254100 & 0.02537700 \\ \mathrm{~N} & -2.35960200 & -0.87582600 & -0.04157600 \\ \mathrm{H} & -2.90171200 & -1.73854000 & -0.09609200\end{array}$




$\begin{array}{lrrr}\text { IM26 } & & & \\ \mathrm{C} & -1.24781700 & 1.12207400 & -0.12457900 \\ \mathrm{C} & -0.21122300 & 0.25471400 & 0.25658100 \\ \mathrm{H} & -1.24266700 & 2.19379200 & -0.25102100 \\ \mathrm{~N} & 2.02585900 & -0.25344300 & -0.47093600 \\ \mathrm{O} & 3.11882100 & -0.14861800 & -0.14773800 \\ \mathrm{O} & 1.06526700 & 0.54714700 & 0.55985200 \\ \mathrm{~N} & -0.68517300 & -1.01286500 & 0.29847600 \\ \mathrm{~N} & -2.36916200 & 0.39546800 & -0.30659800 \\ \mathrm{~N} & -1.95539100 & -0.84520500 & -0.05265500 \\ \mathrm{H} & -2.58873100 & -1.63044500 & -0.11591300\end{array}$




\section{Products:}

P1

$\begin{array}{llll}\mathrm{C} & 1.08269900 & 0.25808700 & -0.00047800\end{array}$

$\begin{array}{llll}\mathrm{H} & 0.00082800 & 2.10111600 & -0.00256800\end{array}$

$\begin{array}{llll}\mathrm{H} & 2.10687200 & 0.60017500 & 0.00068400\end{array}$

$\mathrm{N} \quad 0.68934100 \quad-1.00345800 \quad 0.00007700$

C $\quad-1.08248000 \quad 0.25891200 \quad-0.00034900$

$\mathrm{N} \quad-0.69014200 \quad-1.00294600 \quad-0.00001100$

$\begin{array}{llll}\mathrm{H} & -2.10644400 & 0.60181200 & 0.00048700\end{array}$

$\begin{array}{llll}\mathrm{N} & 0.00043400 & 1.09139100 & 0.00084200\end{array}$

P2

$\begin{array}{llll}\mathrm{C} & 2.48608200 & -0.84009200 & -0.08729500\end{array}$

$\mathrm{H} \quad-1.16390700 \quad 0.37767800 \quad-0.05206400$

$\mathrm{H} \quad 2.89090600 \quad-1.72405400 \quad 0.36274300$

$\begin{array}{llll}\mathrm{N} & 1.72784500 & 0.06286200 & 0.03120000\end{array}$

C $\quad-2.17830000 \quad 0.00894800 \quad-0.01873600$

$\mathrm{N} \quad \begin{array}{llll}\mathrm{N} & 0.85971800 & 0.97955700 & -0.05972800\end{array}$

$\mathrm{H} \quad \begin{array}{llll}\mathrm{H} & 1.24895000 & 1.82500100 & 0.36480000\end{array}$

$\mathrm{N} \quad-3.27651200 \quad-0.39838500 \quad 0.02291300$

P3

$\begin{array}{llll}\mathrm{C} & 1.13677000 & 1.30292600 & 0.00677200\end{array}$

$\mathrm{H} \quad 0.96260300 \quad-1.73232400 \quad 0.86563200$

$\mathrm{H} \quad \begin{array}{llll}\mathrm{H} & 1.93487700 & 2.00518500 & 0.24342800\end{array}$

$\mathrm{N} \quad-2.04542500 \quad 0.54615700 \quad-0.12524200$

C $\quad \begin{array}{llll}\mathrm{C} & 1.23346100 & -1.19588800 & -0.03344800\end{array}$

$\begin{array}{llll}\mathrm{N} & -1.89089100 & -0.53799200 & 0.11841300\end{array}$

$\mathrm{H} \quad 1.46127900 \quad-1.69151000 \quad-0.96744200$

$\mathrm{N} \quad \begin{array}{llll}1.28200900 & 0.10275300 & 0.00946300\end{array}$

P4

C $\quad 2.04481000 \quad-0.90662400 \quad-0.34555500$

$\begin{array}{llll}\mathrm{H} & 0.60073900 & 0.74502700 & -1.20397700\end{array}$

$\mathrm{H} \quad 2.72044300 \quad-1.44623800 \quad 0.35119800$

$\begin{array}{lrrr}\mathrm{H} & 1.97432500 & 0.40001800 & 1.28006200\end{array}$

$\begin{array}{llll}\mathrm{N} & -1.87645100 & -0.26835700 & 0.02672800\end{array}$

$\begin{array}{llll}\mathrm{N} & -2.98511400 & -0.10780200 & 0.04885000\end{array}$

$\begin{array}{llll}\mathrm{C} & 0.81473600 & 1.15337700 & -0.19946300\end{array}$

$\begin{array}{llll}\mathrm{N} & 1.65402500 & 0.20768400 & 0.33054000\end{array}$

P5

$\begin{array}{llll}\text { C } & -1.85966700 & -0.59632000 & -0.15021400\end{array}$

$\mathrm{H} \quad 1.83872700 \quad-1.58112400 \quad 0.75434000$

$\mathrm{H} \quad-1.72783200 \quad-1.60569800 \quad-0.47557200$

$\begin{array}{llll}\mathrm{H} & 0.31347500 & 1.75119100 & 0.22831600\end{array}$

$\mathrm{N} \quad \begin{array}{llll}0.33157200 & -1.15494900 & -0.01505000\end{array}$

$\begin{array}{llll}\mathrm{N} & -1.97957600 & 0.51483000 & 0.20153600\end{array}$

$\begin{array}{llll}\mathrm{C} & 1.21890700 & 0.07722100 & 0.02481200\end{array}$

$\begin{array}{lllll}\mathrm{N} & 1.13660200 & 1.29015200 & -0.15144000\end{array}$ 


$\begin{array}{lrrr}\text { P6 } & & & \\ \text { C } & 1.13351500 & 1.29678800 & 0.00015100 \\ \text { H } & 1.18066200 & -1.71910200 & 0.94993000 \\ \text { H } & 1.96347600 & 2.00164400 & -0.00173700 \\ \text { H } & 1.17687500 & -1.71889300 & -0.94970900 \\ \text { N } & -1.88527700 & -0.54365900 & -0.00042900 \\ \text { N } & -2.00790900 & 0.57189000 & 0.00050900 \\ \text { C } & 1.20440100 & -1.20257200 & 0.00010300 \\ \text { N } & 1.27197000 & 0.09620600 & -0.00008100\end{array}$

P7

$\begin{array}{lrrr}\mathrm{C} & 1.31717700 & 0.40250700 & 0.00001700 \\ \mathrm{C} & 1.78973100 & -0.98138100 & -0.00050000 \\ \mathrm{~N} & -1.78610200 & -0.59371900 & 0.00185200 \\ \mathrm{H} & 2.39611900 & -1.16681300 & -0.88904800 \\ \mathrm{H} & 2.39492800 & -1.16792700 & 0.88862800 \\ \mathrm{H} & 0.93400200 & -1.65907000 & -0.00148000 \\ \mathrm{~N} & -2.62428800 & 0.14979700 & -0.00158500 \\ \mathrm{~N} & 0.92946200 & 1.51064400 & 0.00041800\end{array}$

P8

$\begin{array}{llll}\mathrm{C} & -1.57521400 & -1.15812800 & -0.07535700\end{array}$

$\begin{array}{llll}\mathrm{N} & 1.84363100 & -0.45542800 & 0.36313500\end{array}$

$\begin{array}{llll}\mathrm{H} & -1.02763500 & -1.83141400 & 0.57138200\end{array}$

$\begin{array}{llll}\mathrm{H} & -1.57703700 & 1.92305600 & 0.61814000\end{array}$

$\begin{array}{llll}\mathrm{H} & -2.31301100 & -1.54356000 & -0.76688700\end{array}$

$\begin{array}{llll}\mathrm{N} & 2.41897300 & 0.20124300 & -0.33931500\end{array}$

$\mathrm{N} \quad \begin{array}{llll}\mathrm{N} & -1.04410300 & 1.33378700 & -0.02330900\end{array}$

P9

$\begin{array}{lrrr}\mathrm{C} & -1.37463100 & 1.16753400 & 0.00905700 \\ \mathrm{C} & 1.92732700 & 0.58120100 & -0.16766600 \\ \mathrm{H} & -1.83184200 & 1.94940800 & 0.58611000 \\ \mathrm{H} & 1.96114900 & 1.58122800 & -0.54231400 \\ \mathrm{~N} & -1.23508000 & -0.01457400 & 0.02786700 \\ \mathrm{~N} & -1.11736800 & -1.24974300 & -0.17018100 \\ \mathrm{H} & -0.22933800 & -1.54064300 & 0.25618700 \\ \mathrm{~N} & 1.89299900 & -0.51888300 & 0.23540900\end{array}$

P10

$\begin{array}{lrrr}\mathrm{C} & -0.17003800 & 1.13336800 & 0.00065300 \\ \mathrm{C} & 1.05120700 & 0.48083700 & -0.00027400 \\ \mathrm{H} & -0.46000900 & 2.17226300 & -0.00161200 \\ \mathrm{H} & 2.04747500 & 0.89602800 & -0.00051300 \\ \mathrm{~N} & -1.06417900 & 0.11312500 & -0.00021400 \\ \mathrm{~N} & -0.46301800 & -1.09224900 & -0.00034300 \\ \mathrm{H} & -2.07430200 & 0.15259000 & 0.00108700 \\ \mathrm{~N} & 0.84145700 & -0.86460600 & 0.00038100\end{array}$

P11

$\mathrm{C}$

$\begin{array}{llll}\mathrm{H} & 1.69210200 & 1.30263100 & 0.00008300\end{array}$

$\begin{array}{lllll}\mathrm{N} & -0.37047000 & 1.07420900 & -0.00023100\end{array}$

C $\quad-0.23835600-1.10070900-0.00002100$

$\begin{array}{llll}\mathrm{N} & -1.09148400 & -0.10324400 & 0.00012500\end{array}$

$\mathrm{H} \quad-2.10124000 \quad-0.09622400 \quad 0.00012100$

$\mathrm{N} \quad \begin{array}{llll}1.00000800 & -0.74040000 & -0.00012200\end{array}$ 


$\begin{array}{lrrr}\text { P12 } & & & \\ \text { C } & -2.43902800 & 0.18068900 & -0.13941900 \\ \text { H } & -3.23000900 & 0.54509100 & 0.50182300 \\ \text { C } & -0.16716300 & -0.82749200 & 0.02826000 \\ \text { H } & 0.02305800 & -1.88812900 & -0.00387600 \\ \text { N } & -1.37201600 & -0.31956700 & 0.02765200 \\ \text { N } & 0.92791900 & 0.08054600 & 0.00516900 \\ \text { O } & 0.68953800 & 1.29523500 & 0.00312700 \\ \text { O } & 2.05455800 & -0.43310900 & -0.01072000 \\ & & & \\ \text { P13 } & & & \\ \text { C } & 1.26755200 & -0.65671200 & -0.00006100 \\ \text { H } & 2.20547300 & -1.20079400 & 0.00006800 \\ \text { N } & 1.33799600 & 0.75226400 & -0.00079800 \\ \text { C } & -0.78527500 & -0.13087000 & 0.00026300 \\ \text { N } & 0.09526300 & 1.05236500 & 0.00113100 \\ \text { H } & -0.21621100 & 2.01940500 & -0.00118500 \\ \text { N } & 0.11838100 & -1.19526500 & 0.00022200 \\ \text { O } & -1.96805100 & -0.04483200 & -0.00049800\end{array}$

$\begin{array}{lrcc}\text { P14 } & & & \\ \text { C } & 0.80800500 & 0.65146900 & 0.00000700 \\ \text { H } & 1.57596300 & 1.41420200 & 0.00004600 \\ \text { N } & -0.52253000 & 0.97322000 & 0.00010000 \\ \text { C } & -0.19192700 & -0.85943300 & -0.00005900 \\ \text { N } & -1.31871900 & -0.28351100 & -0.00011800 \\ \text { N } & 1.08804500 & -0.71348300 & 0.00005500\end{array}$

$\begin{array}{lrrr}\text { P15 } & & & \\ \text { C } & 2.01575900 & 0.61762200 & -0.10695200 \\ \text { H } & 2.91823600 & 1.20738200 & -0.20291100 \\ \text { N } & 2.05865600 & -0.67767800 & 0.30822800 \\ \text { C } & 0.03888500 & 0.00457000 & -0.18937300 \\ \text { N } & 0.80331900 & -1.12130800 & 0.35220000 \\ \text { H } & 0.28363000 & -1.03893900 & -0.86386700 \\ \text { N } & 0.80059000 & 1.08277600 & -0.42963800 \\ \text { N } & -1.41644800 & 0.04556900 & -0.00465200 \\ \text { O } & -2.03439100 & -0.95528100 & -0.36717100 \\ \text { O } & -1.87217800 & 1.05439200 & 0.52489100\end{array}$

P16

$\begin{array}{lrrl}\mathrm{C} & 0.70492100 & 0.77299500 & 0.00028900 \\ \mathrm{H} & 1.32406000 & 1.66038700 & -0.00046400 \\ \mathrm{~N} & 1.14510300 & -0.48578700 & -0.00047700 \\ \mathrm{~N} & -1.12031900 & -0.47568800 & -0.00056800 \\ \mathrm{~N} & -0.61832100 & 0.80517500 & 0.00008400 \\ \mathrm{H} & -1.24623700 & 1.59872500 & 0.00059700 \\ \mathrm{C} & -0.02543100 & -1.13383000 & 0.00081000\end{array}$

P17

$\begin{array}{lrrr}\mathrm{C} & -2.41706700 & -0.00017900 & -0.33774400 \\ \mathrm{H} & -2.89398300 & 0.94796300 & -0.56356400 \\ \mathrm{~N} & -1.26035800 & 0.00030500 & 0.19914700 \\ \mathrm{H} & -2.89409000 & -0.94883200 & -0.56128700 \\ \mathrm{C} & -0.22288600 & 0.00118000 & 0.87282200 \\ \mathrm{~N} & 0.99600500 & -0.00011300 & 0.03734600 \\ \mathrm{O} & 1.46686000 & -1.10021200 & -0.23343200 \\ \mathrm{O} & 1.46792400 & 1.09940200 & -0.23420200\end{array}$




$\begin{array}{lrrr}\text { P18 } & & & \\ \text { C } & 1.20976100 & 0.73476300 & 0.00000200 \\ \text { H } & 2.12879900 & 1.31190100 & -0.00006500 \\ \text { C } & -0.81014400 & 0.02477500 & 0.00008900 \\ \text { N } & 0.02303000 & 1.19744100 & 0.00013900 \\ \text { O } & -1.98555600 & -0.03438700 & -0.00014100 \\ \text { N } & 0.04850300 & -1.16266100 & 0.00023700 \\ \text { N } & 1.25534400 & -0.65464700 & -0.00040400 \\ \text { H } & 2.06980800 & -1.25496200 & 0.00085000 \\ & & & \\ \text { P19 } & & & \\ \text { C } & -0.65092300 & 0.82279600 & -0.00011600 \\ \text { H } & -1.38084200 & 1.62043500 & -0.00023600 \\ \text { H } & -1.90283700 & -0.93626600 & -0.00035600 \\ \text { N } & 1.19924600 & -0.32270700 & 0.00019500 \\ \text { N } & 0.63133700 & 0.97354800 & 0.00016600 \\ \text { C } & 0.21569400 & -1.10495900 & 0.00000800 \\ \text { N } & -0.98843100 & -0.50672500 & -0.00018400\end{array}$

P20

$\begin{array}{lrrr}\mathrm{C} & 2.41753200 & -0.00006500 & -0.33745400 \\ \mathrm{H} & 2.89454600 & 0.94833200 & -0.56201300 \\ \mathrm{H} & 2.89467400 & -0.94850800 & -0.56151000 \\ \mathrm{C} & 0.22285100 & 0.00019400 & 0.87205000 \\ \mathrm{~N} & 1.26054900 & 0.00003100 & 0.19891100 \\ \mathrm{~N} & -0.99614000 & -0.00003300 & 0.03746200 \\ \mathrm{O} & -1.46765100 & 1.09979000 & -0.23371900 \\ \mathrm{O} & -1.46764700 & -1.09986200 & -0.23361300\end{array}$

P21

$\mathrm{C}$

$\begin{array}{lll}1.32292800 & 0.56737000 & 0.00012100\end{array}$

$\begin{array}{llll}\mathrm{H} & 2.23340500 & 1.15314000 & 0.00094400\end{array}$

$\begin{array}{llll}\mathrm{H} & -0.18186100 & 2.03623500 & 0.00233600\end{array}$

$\begin{array}{llll}\mathrm{N} & 0.08143800 & -1.18005300 & -0.00005100\end{array}$

$\begin{array}{llll}\mathrm{N} & 1.31490400 & -0.77484900 & 0.00009900\end{array}$

$\begin{array}{llll}\mathrm{C} & -0.78495900 & -0.06616300 & -0.00001500\end{array}$

$\begin{array}{llll}\mathrm{N} & 0.10840300 & 1.06780300 & -0.00082300\end{array}$

$\begin{array}{llll}\mathrm{O} & -1.97657200 & 0.00163600 & 0.00018800\end{array}$

P22

$\begin{array}{lrrr}\mathrm{C} & 0.80733000 & 0.65208900 & 0.00009100 \\ \mathrm{H} & 1.57499400 & 1.41508200 & 0.00016200 \\ \mathrm{~N} & -1.31834600 & -0.28416200 & -0.00010800 \\ \mathrm{~N} & -0.52336600 & 0.97275500 & -0.00003700 \\ \mathrm{C} & -0.19119600 & -0.85961400 & -0.00003300 \\ \mathrm{~N} & 1.08859700 & -0.71286900 & 0.00007200\end{array}$

P23

$\begin{array}{llll}\mathrm{C} & -1.81699400 & -0.47385500 & -0.00010800\end{array}$

$\begin{array}{llll}\mathrm{H} & -2.81095800 & -0.90239900 & -0.00014900\end{array}$

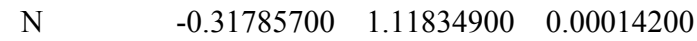

$\begin{array}{llll}\mathrm{N} & -1.62372400 & 0.91679900 & -0.00007300\end{array}$

C $\quad 0.20170200 \quad-0.16057900-0.00000800$

$\mathrm{N} \quad-0.69793300 \quad-1.18261400 \quad 0.00016400$

$\mathrm{N} \quad \begin{array}{llll}\mathrm{N} & 1.53346300 & -0.40231100 & -0.00010100\end{array}$

$\begin{array}{llll}\mathrm{O} & 2.53063300 & 0.19468000 & -0.00001000\end{array}$ 


$\begin{array}{lccc}\text { P24 } & & & \\ \text { C } & 1.12058600 & 0.37040900 & -0.00002300 \\ \text { C } & 0.01671200 & 1.14903500 & 0.00002600 \\ \text { N } & -1.02496200 & 0.36610500 & 0.00002600 \\ \text { H } & -2.01397400 & 0.58514000 & -0.00019200 \\ \text { H } & 2.16830900 & 0.60975600 & -0.00005900 \\ \text { N } & -0.60992700 & -0.92624900 & -0.00000600 \\ \text { N } & 0.63801300 & -0.91293600 & 0.00001300\end{array}$

$\begin{array}{lrrr}\text { P25 } & & & \\ \text { C } & 1.49635400 & -0.20148600 & -0.00164300 \\ \text { C } & 0.22881100 & -0.81056300 & 0.38519100 \\ \text { H } & 0.07049800 & -1.76479600 & -0.11673500 \\ \text { H } & 0.17822500 & -0.93953100 & 1.46905000 \\ \text { N } & 2.53631700 & 0.24732300 & -0.31262500 \\ \text { N } & -0.93262300 & 0.08565100 & 0.01456700 \\ \text { O } & -1.94078200 & -0.48307100 & -0.39491100 \\ \text { O } & -0.78741400 & 1.28879700 & 0.19901200\end{array}$

P26

$\begin{array}{lrrr}\mathrm{C} & -0.22110300 & 1.12917200 & 0.00015200 \\ \mathrm{C} & 0.79957500 & 0.06122900 & 0.00000900 \\ \mathrm{~N} & -0.06201000 & -1.04018000 & 0.00041400 \\ \mathrm{H} & 0.19673900 & -2.01909200 & -0.00092100 \\ \mathrm{H} & -0.02672600 & 2.19223000 & 0.00032100 \\ \mathrm{~N} & -1.33400800 & -0.70022800 & -0.00008500 \\ \mathrm{~N} & -1.41820200 & 0.62974700 & -0.00021200 \\ \mathrm{O} & 2.00733700 & 0.05738600 & -0.00014800\end{array}$

\section{P27}

$\begin{array}{llll}\mathrm{C} & 1.10564000 & 0.50408700 & 0.00005400\end{array}$

$\begin{array}{lllll}\mathrm{C} & -0.20074200 & 0.92943800 & -0.00006300\end{array}$

$\begin{array}{llll}\mathrm{N} & -1.32910200 & 0.34468200 & 0.00002500\end{array}$

$\begin{array}{llll}\mathrm{H} & 2.05312000 & 1.01285100 & 0.00002100\end{array}$

$\begin{array}{llll}\mathrm{N} & -0.58048900 & -0.90602600 & 0.00001300\end{array}$

$\begin{array}{llll}\mathrm{N} & 0.84066200 & -0.81208500 & -0.00003300\end{array}$

\section{P28}

$\begin{array}{lrrr}\mathrm{C} & 0.46933200 & 1.11832700 & 0.00022300 \\ \mathrm{C} & -0.23769400 & -0.11185200 & -0.00008900 \\ \mathrm{~N} & 0.67762700 & -1.16636200 & 0.00001600 \\ \mathrm{H} & 0.09692600 & 2.13240300 & 0.00031200 \\ \mathrm{~N} & 1.83688300 & -0.63741900 & -0.00023300 \\ \mathrm{~N} & 1.76111600 & 0.80787600 & -0.00005900 \\ \mathrm{~N} & -1.55737700 & -0.40052800 & 0.00070400 \\ \mathrm{O} & -2.56431300 & 0.20047300 & -0.00051400\end{array}$

\section{P29}

$\begin{array}{lrrr}\mathrm{C} & -0.60169200 & 0.94023600 & 0.18086500 \\ \mathrm{C} & -1.57109300 & 0.04027300 & 0.06054400 \\ \mathrm{~N} & -2.53023200 & -0.69446200 & -0.19594500 \\ \mathrm{H} & -2.65720100 & -1.46135900 & 0.46905800 \\ \mathrm{H} & -0.74240400 & 1.83744200 & 0.77301100 \\ \mathrm{~N} & 1.38748200 & -0.36956000 & 0.38155700 \\ \mathrm{O} & 2.41589300 & -0.57775700 & -0.11145200 \\ \mathrm{O} & 0.63855200 & 0.72638500 & -0.38727300\end{array}$




$\begin{array}{lccc}\text { P30 } & & & \\ \text { C } & -0.91861500 & 0.00031700 & -0.00012500 \\ \text { N } & 0.50548300 & 0.00008400 & 0.00002200 \\ \text { O } & 1.04079000 & 1.10548300 & 0.00001000 \\ \text { O } & 1.03967500 & -1.10602700 & 0.00001000 \\ \text { N } & -2.09577200 & 0.00026500 & 0.00006300 \\ & & & \\ \text { C } & -1.15967700 & 0.00023600 & -0.05471100 \\ \text { H } & -1.63279500 & -0.95303500 & 0.12324000 \\ \text { N } & 1.30090600 & 0.00033000 & 0.00105700 \\ \text { N } & 0.15936100 & -0.00073000 & 0.01063300 \\ \text { H } & -1.63101800 & 0.95441100 & 0.12319800\end{array}$

\section{P31}

$\begin{array}{lrrr}\mathrm{C} & -1.15966300 & 0.73698900 & 0.00045100 \\ \mathrm{C} & 0.16674900 & 0.95029400 & -0.00001100 \\ \mathrm{~N} & 0.57326100 & -0.93780900 & 0.00068900 \\ \mathrm{~N} & 1.26024200 & 0.29897700 & -0.00042400 \\ \mathrm{~N} & -0.77893100 & -0.60813000 & -0.00050700 \\ \mathrm{H} & -1.42451700 & -1.39495500 & -0.00095100\end{array}$

\section{P32}

$\begin{array}{lrrr}\mathrm{C} & 0.75866000 & -1.26176900 & -0.00003100 \\ \mathrm{C} & -0.22900900 & -0.18794300 & 0.00000600 \\ \mathrm{~N} & 1.52869600 & 0.94618300 & 0.00001000 \\ \mathrm{~N} & -1.55794500 & -0.42590700 & 0.00002300 \\ \mathrm{O} & -2.54325900 & 0.18194700 & 0.00001400 \\ \mathrm{~N} & 0.27660500 & 1.06371500 & -0.00004300 \\ \mathrm{~N} & 1.80713700 & -0.44821700 & 0.00001100 \\ \mathrm{H} & 2.78672000 & -0.70773100 & 0.00002800\end{array}$

\section{P33}

$\begin{array}{lrrr}\mathrm{C} & 1.25950400 & 0.40197000 & -0.11157500 \\ \mathrm{C} & -0.21170200 & 0.60827900 & 0.07566400 \\ \mathrm{H} & 1.81018500 & 1.29734500 & -0.41920100 \\ \mathrm{~N} & -0.99567200 & -0.57724100 & 0.54241600 \\ \mathrm{O} & -1.24357800 & -1.31989600 & -0.39878300 \\ \mathrm{O} & -0.77872100 & 1.66369700 & -0.07528100 \\ \mathrm{~N} & 1.75508700 & -0.76053700 & 0.09906900 \\ \mathrm{H} & 2.76548600 & -0.74480000 & -0.06322500\end{array}$

\section{P34}

$\begin{array}{lrrr}\mathrm{C} & 1.15341400 & 0.15151400 & 0.00007100 \\ \mathrm{C} & 0.16994700 & 1.15660300 & -0.00016500 \\ \mathrm{H} & 2.22877600 & 0.19961000 & 0.00001200 \\ \mathrm{~N} & -0.99377900 & 0.65410800 & 0.00007000 \\ \mathrm{~N} & 0.50085100 & -0.97763000 & -0.00003900 \\ \mathrm{~N} & -0.74683700 & -0.63693300 & 0.00003600 \\ \mathrm{H} & -1.49058800 & -1.32512400 & 0.00008900\end{array}$




$\begin{array}{lrrr}\text { P35 } & & & \\ \text { C } & 0.89934300 & 1.15059600 & 0.00000000 \\ \text { C } & 0.00805200 & 0.08970900 & 0.00005700 \\ \text { N } & 0.77592300 & -1.01746200 & 0.00025300 \\ \text { H } & 0.47533800 & -1.98615400 & 0.00045100 \\ \text { H } & 0.69973500 & 2.21081200 & -0.00007100 \\ \text { N } & 2.08357700 & -0.69390500 & 0.00003500 \\ \text { N } & 2.15325000 & 0.63304500 & -0.00020200 \\ \text { N } & -1.42942700 & 0.01700300 & 0.00003000 \\ \text { O } & -1.92394300 & -1.11384700 & 0.00002600 \\ \text { O } & -2.03889500 & 1.08419100 & -0.00021900\end{array}$

$\begin{array}{lrrc}\text { P36 } & & & \\ \text { C } & -0.82820400 & 1.10026700 & 0.00017500 \\ \text { C } & -0.00668200 & -0.01185400 & 0.00001000 \\ \text { H } & -0.63936300 & 2.16136900 & 0.00028400 \\ \text { N } & -2.00558600 & -0.81409400 & -0.00002900 \\ \text { N } & 1.44667100 & 0.00156700 & -0.00004200 \\ \text { O } & 2.03318100 & -1.07513000 & -0.00018600 \\ \text { O } & 1.96741100 & 1.11955700 & 0.00002000 \\ \text { N } & -0.72577400 & -1.15329200 & -0.00004000 \\ \text { N } & -2.05711200 & 0.53264100 & 0.00008500 \\ \text { H } & -2.96344500 & 0.98498700 & 0.00012300\end{array}$

$\begin{array}{lrrr}\text { P37 } & & & \\ \text { C } & -0.13758700 & 1.13344100 & -0.00019200 \\ \text { C } & 0.80599700 & -0.02367600 & 0.00023700 \\ \text { H } & 0.12011400 & 2.18260600 & -0.00080600 \\ \text { O } & 2.00963700 & -0.03931800 & -0.00010300 \\ \text { N } & -0.04501900 & -1.13593700 & 0.00023400 \\ \text { N } & -1.33832600 & 0.72657600 & 0.00045600 \\ \text { N } & -1.21125000 & -0.63545300 & -0.00039700 \\ \text { H } & -2.04550100 & -1.21295600 & -0.00069300\end{array}$




\section{Small Molecules:}

$\mathrm{N}_{2}$

$\begin{array}{llll}\mathrm{N} & 0.00000000 & 0.00000000 & 0.56013400\end{array}$

$\begin{array}{llll}\mathrm{N} & 0.00000000 & 0.00000000 & -0.56013400\end{array}$

$\mathrm{NO}_{2}$

$\begin{array}{llll}\mathrm{N} & 0.00000000 & 0.32747900 & 0.00000000\end{array}$

$\begin{array}{llll}\mathrm{O} & 1.10690300 & -0.14329500 & 0.00000000\end{array}$

$\begin{array}{llll}\mathrm{O} & -1.10690300 & -0.14324900 & 0.00000000\end{array}$

NO

$\begin{array}{llll}\mathrm{N} & 0.00000000 & 0.00000000 & -0.60509000\end{array}$

$\begin{array}{llll}\mathrm{O} & 0.00000000 & 0.00000000 & 0.52945400\end{array}$

\section{HONO}

$\begin{array}{llll}\mathrm{H} & 1.70879800 & 0.44035800 & 0.00011600\end{array}$

$\begin{array}{llll}\mathrm{N} & -0.16744600 & 0.48920800 & 0.00001900\end{array}$

$\begin{array}{llll}\mathrm{O} & -1.10681000 & -0.22357400 & -0.00009900\end{array}$

$\begin{array}{llll}\mathrm{O} & 1.03972600 & -0.25952800 & 0.00006800\end{array}$

$\mathrm{OH}$

$\begin{array}{llll}\mathrm{H} & 0.00000000 & 0.00000000 & -0.86106100\end{array}$

$\begin{array}{llll}\mathrm{O} & 0.00000000 & 0.00000000 & 0.10763300\end{array}$ 